\title{
Simulation-Based Resilience Quantification of an Indoor Ultrasound Localization System in the Presence of Disruptions
}

\author{
Aishvarya Kumar Jain ${ }^{1, *(\mathbb{D})}$, Dominik Jan Schott ${ }^{2}{ }^{(\mathbb{D}}$, Hermann Scheithauer ${ }^{3}$, Ivo Häring ${ }^{1}{ }^{(\mathbb{D}}$, \\ Fabian Höflinger ${ }^{1}{ }^{1}$, Georg Fischer ${ }^{1} \mathbb{D}$, Emanuël A. P. Habets ${ }^{4} \mathbb{D}^{\mathbb{D}}$, Patrick Gelhausen ${ }^{1}$, \\ Christian Schindelhauer ${ }^{5}$ (D) and Stefan Johann Rupitsch ${ }^{2}$ (i)
}

1 Fraunhofer Institute for High-Speed Dynamics, Ernst-Mach-Institute (EMI), 79588 Efringen-Kirchen, Germany; ivo.haering@emi.fraunhofer.de (I.H.); Fabian.Hoeflinger@emi.fraunhofer.de (F.H.); Georg.Fischer@emi.fraunhofer.de (G.F.); patrick.gelhausen@fernuni-hagen.de (P.G.)

2 Department of Microsystem Engineering (IMTEK), University of Freiburg, 79110 Freiburg, Germany; dominik.jan.schott@imtek.uni-freiburg.de (D.J.S.); stefan.rupitsch@imtek.uni-freiburg.de (S.J.R.)

3 Hahn-Schickard, 78052 Villingen-Schwenningen, Germany; Hermann.Scheithauer@hahn-schickard.de

4 International Audio Laboratories Erlangen, University of Erlangen-Nuremberg, 91058 Erlangen, Germany; emanuel.habets@audiolabs-erlangen.de

5 Department of Computer Science (IIF), University of Freiburg, 79110 Freiburg, Germany; schindel@informatik.uni-freiburg.de

* Correspondence: aishvarya.kumar.jain@emi.fraunhofer.de

Citation: Jain, A.K.; Schott, D.J.; Scheithauer, H.; Häring, I.; Höflinger, F.; Fischer, G.; Habets, E.A.P.; Gelhausen, P.; Schindelhauer, C.; Rupitsch, S.J.; Simulation-Based Resilience Quantification of an Indoor Ultrasound Localization System in the Presence of Disruptions. Sensors 2021, 21, 6332. https://doi. org/10.3390/s21196332

Academic Editors: Antonio Moschitta and Joanna Kolodziej

Received: 22 June 2021

Accepted: 16 September 2021

Published: 22 September 2021

Publisher's Note: MDPI stays neutral with regard to jurisdictional claims in published maps and institutional affiliations.

Copyright: (c) 2021 by the authors. Licensee MDPI, Basel, Switzerland. This article is an open access article distributed under the terms and conditions of the Creative Commons Attribution (CC BY) license (https:/ / creativecommons.org/licenses/by/ $4.0 /)$.

\begin{abstract}
Time difference of arrival (TDOA) based indoor ultrasound localization systems are prone to multiple disruptions and demand reliable, and resilient position accuracy during operation. In this challenging context, a missing link to evaluate the performance of such systems is a simulation approach to test their robustness in the presence of disruptions. This approach cannot only replace experiments in early phases of development but could also be used to study susceptibility, robustness, response, and recovery in case of disruptions. The paper presents a simulation framework for a TDOA-based indoor ultrasound localization system and ways to introduce different types of disruptions. This framework can be used to test the performance of TDOA-based localization algorithms in the presence of disruptions. Resilience quantification results are presented for representative disruptions. Based on these quantities, it is found that localization with arc-tangent cost function is approximately $30 \%$ more resilient than the linear cost function. The simulation approach is shown to apply to resilience engineering and can be used to increase the efficiency and quality of indoor localization methods.
\end{abstract}

Keywords: indoor localization; simulation; time difference of arrival; disruption; technical resilience; localization accuracy; ultrasound; cross correlation; loss function; resilience engineering

\section{Introduction}

Acoustic indoor localization systems, with applications in logistics, consumer electronics, health care, security, and catastrophe management, are required to be precise and reliable. Furthermore, they should be resilient to expected disruptions [1-4]. Physical testing of these systems during the development phase can incur a huge amount of time, increasing the development and other overhead costs. Simulating the behavior of these systems can reduce time and money. As the performance of these systems is correlated to the operational environment in which they are employed, it is essential to simulate the behavior of the environment. Two of the most important environmental factors that affect the performance of acoustic indoor localization are the geometry of the room and the absence or presence of disruptions. Disruption refers to any process that hinders the normal functionality of the localization system, for example, noise in the environment or malfunctioning receivers. A framework capable of simulating different environments 
and disruptions can be used to simulate the behavior of indoor localization systems and evaluate the performance in different environments and with different disruptions.

The main objective of the paper is to present a systematic methodology to test TDOAbased indoor ultrasound localization systems in a simulation environment before entering the production phase and during product improvement cycles. The aim is to quantify and visualize the localization accuracy and resilience in the presence and absence of disruptions. To achieve this objective, we propose an end-to-end simulation and evaluation framework, which includes a method to perform acoustic source localization and a method to evaluate the system's performance and measure its resilience. The proposed framework can be used to detect the strengths and weaknesses of localization algorithms during the development and testing phase. As an example, we used the proposed framework to test one of the existing iterative TDOA-based localization algorithms, but the same methodology can be applied to test other TDOA-based algorithms. The simulation of the indoor ultrasound propagation is performed for a shoe-box room and can be extended to more complex room geometries. We also demonstrate how this framework can be used to induce multiple disruptions and how the performance of the localization system can be quantified and compared in the presence of such disruptions. In particular, we focused on some basic disruptions due to noise and barriers as well as receiver malfunction. This framework uses an informed method for system improvement in terms of localization accuracy and resilience, which is demonstrated by an improved functionality when using an arc-tangent loss function instead of a linear loss function.

\subsection{Related Work}

Acoustic indoor localization is a widely studied subject, see [5] and the references therein. Different mechanisms, such as time of flight, doppler effect, and phase shift, can be employed. In the present work, we use TDOAs, which are computed based on time of flight information. The location of a source can then be computed from the TDOAs (see, for example, [6]), which remains a subject of ongoing research. Comuniello et al. [7], for example, recently proposed a best linear unbiased estimator for TDOA-based localization, which is more robust against multipath propagation.

Different frequency ranges can be employed for acoustic indoor localization. The BeepBeep localization system [8] uses the frequency range from $1 \mathrm{kHz}$ up to $20 \mathrm{kHz}$. This frequency range also offers the possibility to use smartphones, without extra hardware, for localization $[9,10]$. Using a commercial off-the-shelf component, one can push the frequency range outside the audible domain, as the ASSIST system [11] does.

Previous studies related to indoor localization have adopted a simulation-based approach to assessing the system's performance. In the radio frequency domain, Sapumohotti et al. [12], for example, proposed a simulation test-bed for WiFi-based localization systems. Their motivation was to reduce the development and testing cost by providing a simulated environment to test the WiFi-based localization systems and algorithms. A similar approach was described by Alhammadi et al. [13], simulating the received signal strength in an indoor environment by considering a multiwall path-loss model. They validated the simulated data using an experimental test bench and showed that simulated data can be used to develop and evaluate high-accuracy localization systems.

To produce representative simulation data, we need to model the propagation of sound waves. In enclosed rooms, the propagation can be simulated using geometric methods (e.g., image-source [14-17] and ray-acoustic methods [18-21]), or wave-based methods (e.g., finite element [22] or boundary element methods [23]). When considering ultrasound waves, we can assume that wavelength is much smaller than the dimensions of the enclosed room and the size of most obstacles. In this work, a ray-acoustic method is adopted as it can take into account solid obstacles and exhibits a lower computational complexity compared to wave-based methods.

The operational performance of localization systems vastly depends on the operating environment and the disruptions. The effect of noise on the performance of acoustic indoor 
localization systems has been studied, for example, in [24]. In practice, the operational performance of a localization system depends on the combination of disruptions and not only noise. Therefore, it is important to study the behavior of acoustic indoor localization systems in the presence of several expected disruptions and measure the resilience of the system against these disruptions. However, the domain of simulation including these disruptions and resilience quantification is relatively unexplored.

\subsection{Structure of this Paper}

The remainder of the paper is organized as follows. In Section 2, we discuss the employed acoustic simulation method and explain how the disruptions are modeled. In Section 3, the TDOA-based localization system under test is described. First, we describe the process of computing TDOAs from the generated RIRs and estimating the acoustic source position from the TDOAs. In Section 4, we discuss how to quantify the resilience of a technical system and correlate it with the simulated indoor localization system presented in this paper. In Section 5, we present the results. First, we investigate the localization with individual disruptions and compare the performance of arc-tangent and linear loss functions. Secondly, we evaluate the performance in the presence of multiple disruptions. Finally, we analyze the time-dependent resilience curves, which are used to calculate the resilience of the simulated indoor localization system. In Section 6, we use data collected from real experiments to validate our simulation results. Section 7 presents the conclusion and major findings.

\section{Proposed Simulation Framework}

\subsection{Acoustic Simulation}

In this work, we simulated the indoor ultrasound propagation for all the source and receiver pairs and all frequencies using the ray acoustics method. Each propagation path is modeled by a room impulse response (RIR). A single acoustic source was placed at a random position in the room at a pre-defined height $z_{s}$. In addition, the $N$ receivers were placed randomly in the room at a fixed height $z_{r}$. The frequency range for the simulation was limited to $19-20 \mathrm{kHz}$.

\subsection{Disruption Modeling}

We have included three kinds of disruptions in the simulation framework, namely, barrier in the vicinity of the acoustic source, noise in the environment, and malfunction of the receivers. The disruptions are added individually and also in combination (of two disruptions).

To introduce a barrier into the simulation, a new set of acoustic simulations is performed for each barrier orientation to generate new RIRs specific to the orientation of the barrier.

To introduce noise into the system, the zero-mean Gaussian noise [25] with standard deviation $\sigma$ is directly added to the reverberant signal $g(t)$. The noise is quantified relative to the original signal by comparing the signal-to-noise ratio (SNR) [26]

$$
\mathrm{SNR}_{\mathrm{dB}}=10 \log _{10}\left(\frac{P_{\mathrm{S}}}{P_{\mathrm{N}}}\right),
$$

where $P_{\mathrm{S}}=T^{-1} \int_{0}^{T} g(t)^{2} \mathrm{~d} t$ is the power of the signal $g(t)$ and $P_{\mathrm{N}}=\sigma^{2}$ is the power of the generated noise signal and $T$ is the time period of the signal.

To introduce the effect induced by receiver malfunction, only a subset of receiver instances are used from all the receiver instances. This subset is selected at random from the total set of receiver instances.

\section{Localization System under Test}

The system under test is a TDOA-based indoor ultrasound localization system. In such systems, the clocks of the acoustic source are not synchronized with the clocks of 
acoustic receivers, but clocks of all the acoustic receivers are synchronized together. In such a case, the time of flight of the signal cannot be estimated and thus they resort to the estimation of TDOAs for the localization [27]. The formalization described in the following subsections is used for the implementation of the indoor localization system used in this paper.

\subsection{TDOA Estimation}

For the ultrasound localization system used in this study, linear sine chirps of the form

$$
s(t)=\sin \left(2 \pi\left(f_{0}+\frac{f_{1}-f_{0}}{2 T} t\right) t\right)
$$

are used, because of their high signal-to-noise ratio (SNR) [11]. To generate reverberant signals at each receiver, discrete signal convolution [28] is performed between simulated RIR and the input chirp signal from Equation (2).

The accuracy of the localization system depends on the extraction of correct timestamps, that is, the times when the receivers respectively receive the direct signals, which are termed time of arrival (TOA) timestamps. The TOAs can be extracted through signal cross-correlation between the reverberant signal at each receiver and the original signal [29]. The TOA timestamp is the time where the cross-correlation peaks. Because of reflection, there could be multiple peaks, but the most dominant first peak is created by the direct signal as the direct line of sight between the receiver and source is the shortest path for the signal to travel. If there is an obstruction between the direct line of sight then the dominant peak is generated by the reflected signal. This potentially introduces errors in the source position estimation, but such anomalies could be handled by the loss functions and the minimization method introduced in the Section 3.2. Once TOA timestamps are generated for all the receivers, TDOA timestamps for all receiver source pairs are generated [30],

$$
\begin{gathered}
\mathrm{TDOA}_{\mathrm{ij}}=\mathrm{TOA}_{\mathrm{i}}-\mathrm{TOA}_{\mathrm{j}}, \\
\mathrm{i}, \mathrm{j} \in 1,2, \ldots, N, \\
\mathrm{i} \neq \mathrm{j} .
\end{gathered}
$$

For a system with $N$ receivers, in total $\left(\begin{array}{l}N \\ 2\end{array}\right)$ TDOAs are generated. From the generated TDOAs, the difference between the distance from the source to receiver $i$ and receiver $j$ referred to as the distance difference is calculated using

$$
\Delta d_{\mathrm{ij}}=c \cdot \mathrm{TDOA}_{\mathrm{ij}}
$$

where $c$ is the speed of sound in air.

\subsection{Position Estimation}

The position of the source is calculated using the non-linear least square method with slight modifications [31]. The objective function for the non-linear least square problem is

$$
\begin{aligned}
f\left(\mathbf{q}_{\mathrm{s}} ;\left\{\Delta d_{\mathrm{ij}}\right\} ;\left\{\mathbf{q}_{\mathrm{i}}\right\}\right) & =\sum_{i, j}^{N} \rho\left(\left(\frac{\Delta d_{\mathrm{ij}}-h_{\mathrm{ij}}\left(\mathbf{q}_{\mathrm{s}}\right)}{S}\right)^{2}\right), \\
\text { where } \mathbf{q} & =[x, y, z],
\end{aligned}
$$

where $\mathbf{q}_{\mathrm{s}}$ is the source position, $\left\{\Delta d_{\mathrm{ij}}\right\}$ is the measured distance difference between receivers in Equation (4), $S$ is the scaling factor, and $\left\{\mathbf{q}_{i}\right\}$ is a set with the known receiver positions, with equal and same height $z_{\mathrm{r}}$

$$
\forall i: z_{\mathrm{i}}=z_{\mathrm{r}}
$$


The function $h_{\mathrm{ij}}(\mathbf{q})$ is the expression for the distance difference between receiver $\mathrm{i}$ and receiver $\mathrm{j}$.

$$
h_{\mathrm{ij}}(\mathbf{q})=\left\|\mathbf{q}_{\mathrm{i}}-\mathbf{q}\right\|-\left\|\mathbf{q}_{\mathbf{j}}-\mathbf{q}\right\| .
$$

The function $\rho$ represents the loss function. In this paper, the linear and arc-tangent loss functions [32] are used, i.e.,

$$
\begin{gathered}
\rho_{\operatorname{lin}}(a)=a, \\
\rho_{\mathrm{at}}(a)=\arctan (a) .
\end{gathered}
$$

For given receiver locations $\left\{\mathbf{q}_{i}\right\}$, the non-linear least square estimate of the source coordinates $\hat{\mathbf{q}}_{\mathbf{s}}$ using Equation (5) [31] is

$$
\hat{\mathbf{q}}_{\mathrm{s}}=\arg \min _{x, y} f\left(\mathbf{q}_{\mathrm{s}} ;\left\{\Delta d_{\mathrm{ij}}\right\} ;\left\{\mathbf{q}_{\mathrm{i}}\right\}\right),
$$

which is estimated using an iterative trust-region reflective algorithm [33] as implemented in the Python routine scipy.optimize.least_square (2019). With the rather explicit notation introduced in Equations (3)-(7), Equation (10) is generated and can be used to estimate the source position. Furthermore, other standard approaches to solving the TDOA problem using matrix inversion and iterative matrix equation solvers are documented in the work of Jain [27].

The loss function introduced in Equation (9) can lead to significant improvement in the localization accuracy when disruptions are present in the surrounding environment. The arc-tangent loss function helps to eliminate outliers, which appear when localization is done in the presence of disruptions [34]. The effect is illustrated by comparing the results obtained using the linear and arc-tangent loss functions.

To plot "time-dependent resilience curves", a weighted smoothing with a window length of $N_{\mathrm{w}}$ and weight vector $w_{\mathrm{m}}$ is performed on predicted source position vector at $n$th time index.

$$
\tilde{q}_{\mathbf{s}}(n)=\frac{1}{\sum_{m=0}^{N_{\mathrm{w}}-1} w(m)} \sum_{m=0}^{N_{\mathrm{W}}-1} w(m) q_{\mathrm{s}}(n-m) .
$$

\section{Resilience Quantification}

Resilience quantification of a system is crucial in identifying the vulnerability of the system to various kinds of disruptions. Such disruptions can put the system's functionality to risk. Resilience estimates the functionality of the system in the presence of all representative disruptions. In the case of technical systems, resilience quantification is based on a mathematical formulation of the system's performance. In the current context, it is represented by the localization error, calculated in meters [35]. In the approach shown below, we have extended the resilience methodology to apply in the context of indoor localization systems.

Technical resilience is based on a scalar product of two different vectors: $\mathbf{a}$ and $\mathbf{b}$, which are probabilities of occurrence of each event and cost function of each event, respectively. Both $\mathbf{a}$ and $\mathbf{b}$ are dimensionless non-negative vectors. Given $\mathbf{a}$ and $\mathbf{b}$ the resilience of the system is quantified as,

$$
\mathrm{Rsl}=\exp \left(-\mathbf{a}^{\mathrm{T}} \mathbf{b}\right) .
$$

Figure 1a shows performance behavior for a system. The bright green curve represents the system's response to a rare event, "adaptive behavior", if the disruption has improved the system's performance. The yellow curve is the "ductile behavior", if the performance mildly deteriorates, i.e., the performance stabilizes under a tolerance level. Last, the red curve is the worst case, "collapsing behavior" where the system function breaks down. The dark green curve is the standard case of "robust behavior", where the system reaches the same performance level as before the disruption. 


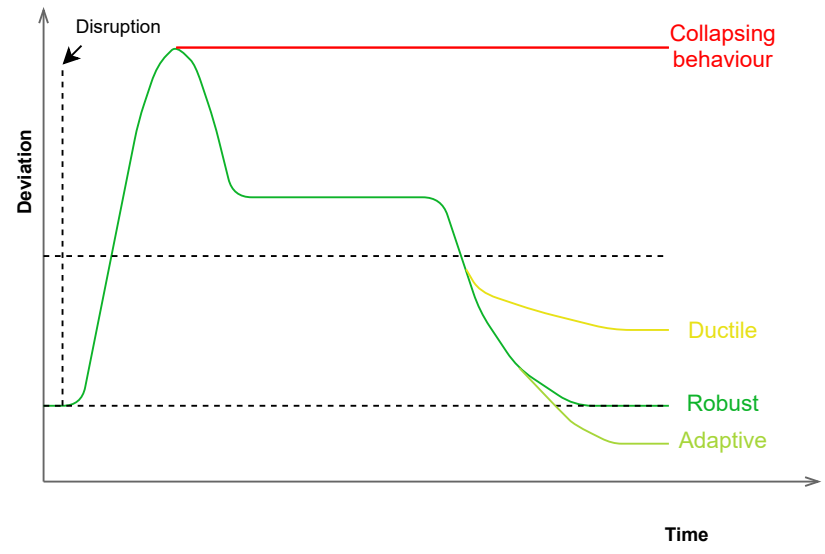

(a)

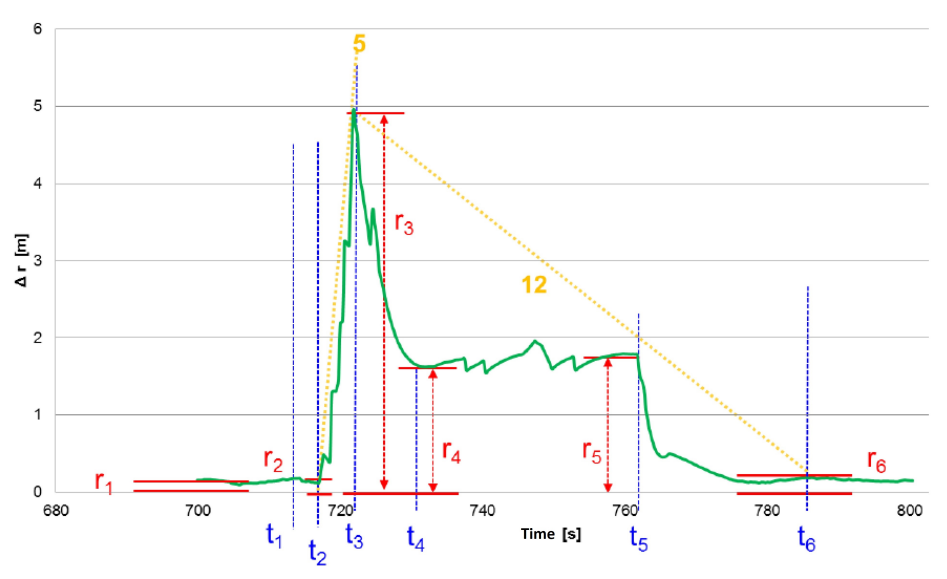

(b)

Figure 1. (a) Different types of performance behavior i.e., collapsing, ductile, robust, and adaptive behavior of a system. (b) Performance behavior of a sample technical system along with corresponding system parameters.

Figure $1 \mathrm{~b}$ represents the robust behavior type of a technical system. The green curve denotes the localization error in meters as a function of time. The representation is termed as "Normally down". Figure $1 \mathrm{~b}$ shows six different errors (in red) which are as follows [36,37]:

- $r_{1}$ : Performance before disruption,

- $r_{2}$ : Performance after delay time,

- $r_{3}$ : Maximum performance loss,

- $r_{4}$ : Temporary performance level, during response phase while disruption continues,

- $r_{5}$ : Performance during the end of disruption, beginning of recovery phase,

- $\quad r_{6}$ : Performance after total recovery.

These errors occur at different times, quantified in seconds (blue markers in Figure 1b) as follows:

- $t_{1}$ : Time of sudden disruption,

- $t_{2}$ : End of delay time, begin of protection phase,

- $t_{3}$ : Time to reach maximum performance loss,

- $t_{4}$ : Time when response-reaction occurs,

- $t_{5}$ : End of disruption, beginning of recovery,

- $t_{6}$ : End of complete recovery, when the pre-disruption state is restored.

The yellow indicators in Figure $1 \mathrm{~b}$ describe the rate of rise and fall of the performance loss from the ground state:

- " 5 ": rate $\left(r_{3}-r_{2}\right) /\left(t_{3}-t_{2}\right)$ describes the rate of maximum performance loss during "protect phase",

- "12": rate $\left(r_{6}-r_{3}\right) /\left(t_{6}-t_{3}\right)$ is the rate of bounce back to the pre-disruption state during "response and recover phase".

Gross et al. [38] describe the optimization of system resilience as a structured $\mathrm{H}_{2}$ norm optimization. It is an integral-quadratic performance criterion that quantifies the system's behaviour after shock. The system behaviour type is like a PD-control. Comparing Figure $1 \mathrm{a}, \mathrm{b}$ the system response is "robust type" between $t_{2}$ and $t_{5}$. Figure 2 shows the quantification of different phases of a resilience curve in terms of loss factor using the indicators described above (except the yellow indicators). The product of the four factors shown in Figure 2 is the "consequence $\left(b_{j}\right)$ ", which is a component of the vector $\mathbf{b}$ in Equation (12), 


$$
b_{\mathrm{j}}=\exp \left[\frac{r_{6 \mathrm{j}}-r_{1 \mathrm{j}}}{R_{0 \mathrm{j}}}\right] \frac{r_{3 \mathrm{j}} r_{4 \mathrm{j}}\left(t_{6 \mathrm{j}}-t_{2 \mathrm{j}}\right)}{R_{0 \mathrm{j}}^{2} T_{0 \mathrm{j}}} \exp \left[\frac{t_{6 \mathrm{j}}-t_{5 \mathrm{j}}}{T_{0 \mathrm{j}}}\right] .
$$

The probability vector a has the component,

$$
a_{\mathrm{j}}=\left[\frac{t_{6 \mathrm{j}}-t_{2 \mathrm{j}}}{T_{\mathrm{tot}}}\right]
$$

which is the quotient between the disruption duration and the total system lifetime $\left(T_{\text {tot }}\right)$.

"Learning" effect: $r_{6}$ vs. $r_{1}$

$$
\exp \left(\frac{r_{6 j}-r_{1 j}}{R_{0 j}}\right)
$$

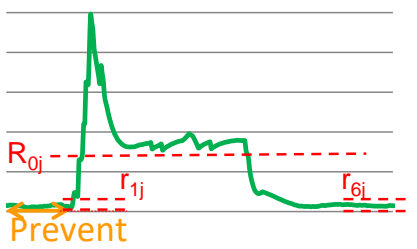

Factors from $\mathrm{H}_{2}$-Norm

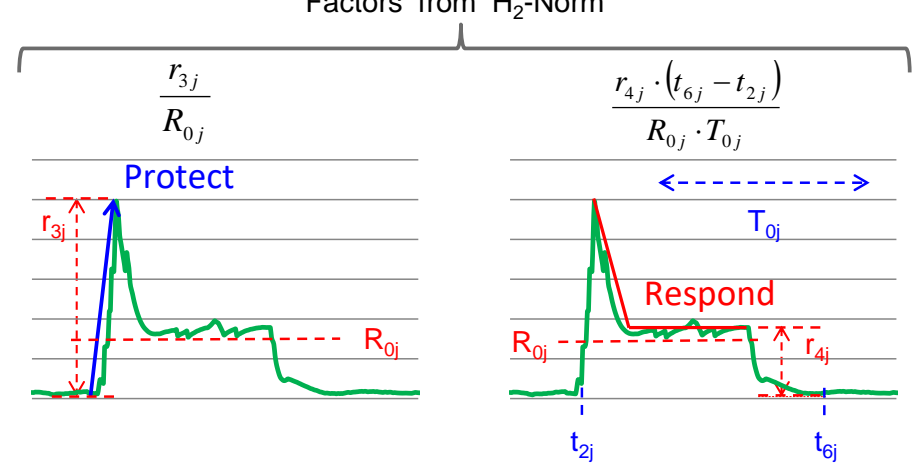

Bounce - Back

$\exp \left(\frac{t_{6 j}-t_{5 j}}{T_{0 j}}\right)$

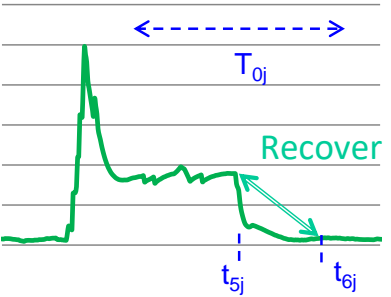

Figure 2. Different phases of a resilience curve showing "Prevent", "Protect", "Respond" and "Recover" phases along with the corresponding loss factor. "Bounce-back" effect refers to the recovery phase of the system.

In Equation (13), the factors $r_{3 j} r_{4 j}\left(t_{6 j}-t_{2 j}\right)$ are an approximation of the quadratic integral. They are scaled by the "Norm performance $\left(R_{0 j}\right)$ " and "Characteristic time $\left(T_{0 j}\right)$ ". These factors only quantifies the "Protect" and "Respond" phases (Figure 2). A single disruption in a robust behaviour also have "Prevent" and "Recover" phases [39-41]. To accommodate these two phases in the resilience quantification, two additional exponential factors (Figure 2) are introduced.

Substitution of Equations (13) and (14) in the Equation (12) leads to the derivation of the resilience of a sample technical system [38] shown in Figure $1 \mathrm{~b}$ as,

$$
\text { Rsl }=\exp \left[-\sum_{j=1}^{H} \frac{t_{6 \mathrm{j}}-t_{2 \mathrm{j}}}{T_{\text {tot }}} \exp \left[\frac{r_{6 \mathrm{j}}-r_{1 \mathrm{j}}}{R_{0 \mathrm{j}}}\right] \frac{r_{3 \mathrm{j}} r_{4 \mathrm{j}}\left(t_{6 \mathrm{j}}-t_{2 \mathrm{j}}\right)}{R_{0 \mathrm{j}}^{2} T_{0 \mathrm{j}}} \exp \left[\frac{t_{6 \mathrm{j}}-t_{5 \mathrm{j}}}{T_{0 \mathrm{j}}}\right]\right],
$$

where $H$ refers to all the disruption events that can affect the functionality of the system and $T_{\text {tot }}$ represent total system's lifetime.

The proposed methodology is applied to the simulated indoor localization system in Section 5. Equations (13) and (14) are used to calculate the elements of $\mathbf{b}$ and a respectively. The corresponding values are then used to estimate the resilience of the system.

\section{Evaluation and Discussion}

The following sections present the localization error plots for linear and arc-tangent cost functions and the resilience quantification of the localization system under test.

\subsection{Setup and Disruptions}

A shoe-box room of dimension $15 \mathrm{~m} \times 15 \mathrm{~m} \times 5 \mathrm{~m}$ which represent a standard office room, is used to demonstrate the proposed approach. The location of the source and receivers is shown in Figure 3, which is one of the many possible constellations. The constellation under test was chosen randomly while keeping in mind that the receivers are spread throughout the dimensions of the room. To simulate the room acoustics, we used the ray acoustics module of COMSOL (version 5.3a) [42], furthermore, we work under the 
assumption that the simulator software provides reliable and realistic results. It is important to mention that this approach can be used to test such a localization system within a much more complex room geometry and with different source-receiver constellations. The speed of sound is assumed to be equal to $343 \mathrm{~m} / \mathrm{s}$.

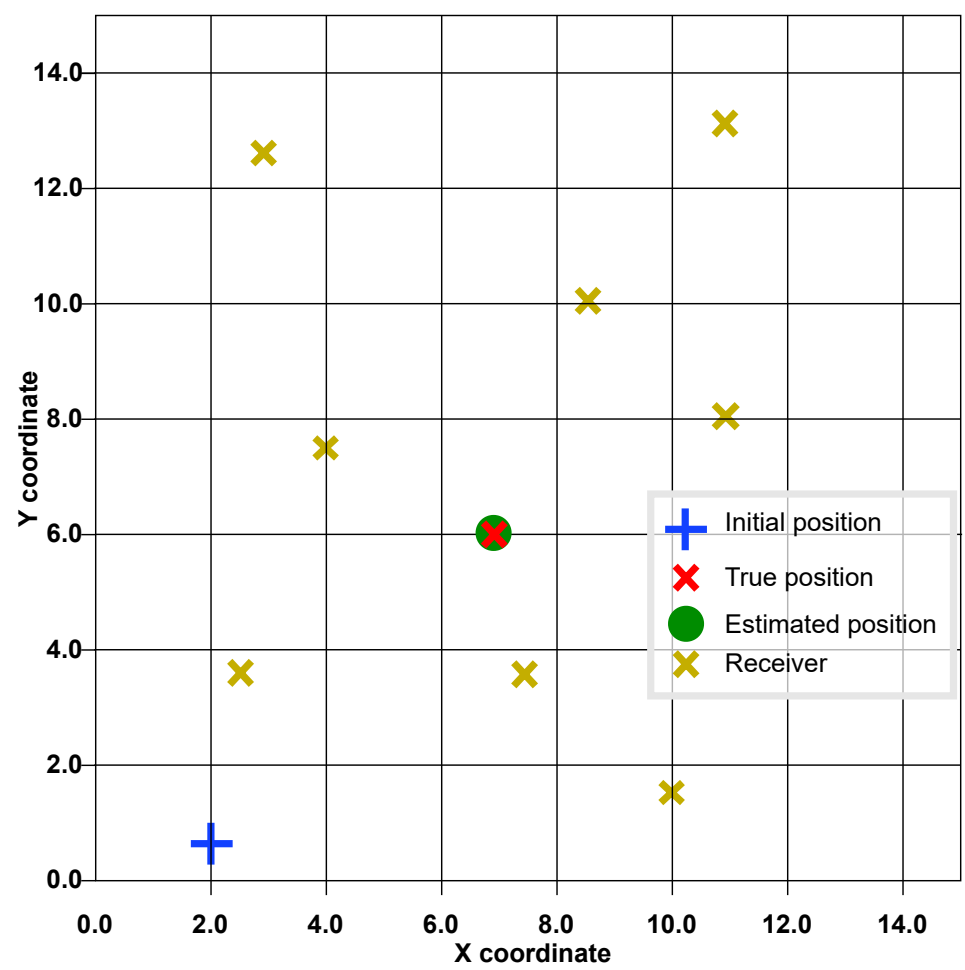

Figure 3. Acoustic simulation setup and implementation verification results showing the final estimated location (see green dot) when the algorithm is initialized with a random location (blue plus in left-bottom corner). In the absence of noise and barriers, and with all the eight receivers (yellow crosses) working, the observed $\Delta r=0.0126 \mathrm{~m}$ (see the red cross for true source position). Room dimensions are $15 \mathrm{~m} \times 15 \mathrm{~m} \times 5 \mathrm{~m}$. Receivers are placed at height $z_{\mathrm{r} 0}=4.9 \mathrm{~m}$ and the source is located at height $z_{\mathrm{s} 0}=1 \mathrm{~m}$.

For barrier as disruption, four different barrier orientations are used as shown in Figure 4.

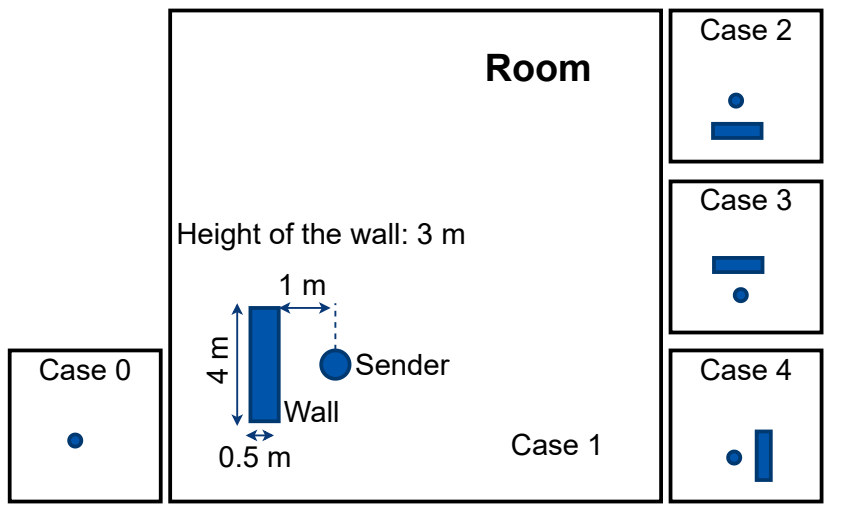

Figure 4. Different orientations of barrier relative to the position of the source representing different cases of barrier disruption. 
For the simulations with increasing noise in the localization environment, $\mu=0$ and $\sigma$ increase in steps of 0.1 from 0 to 1 . This corresponds to $\mathrm{SNR}_{\mathrm{dB}}$ ranging from $\infty$ to $-3 \mathrm{~dB}$ according to Equation (1). Although more simulations were performed with lower $\mathrm{SNR}_{\mathrm{dB}}$, we observed that beyond $-3 \mathrm{~dB} S \mathrm{SN}$, the extracted TOAs are not very accurate, resulting in a very high localization error.

For the simulation with a decreasing number of active receivers, the number of receivers is decreased from eight to three. We believe the upper bound of eight receivers is representative of a room of this dimension. Although, later on, we see the same localization accuracy can also be achieved with fewer receivers, in our study, we decided to use eight receivers.

The localization error plots show different disruption cases along the x-axis and the obtained $\Delta r$ on the y-axis. For the first instance of each simulation, the source position is initialized at random and for later simulated instances, the initialization is done with the final localization estimation of the previous instance. The results are plotted as box plots (bar plot for the barrier as disruption). These curves indicate the robustness of the implemented localization system with respect to the disruptions and compare the performance of linear and arc-tangent loss functions. For each disruption case, 100 source instances (with the same source position) are evaluated and plotted using the box plots. Along with the instances, a mean (black diamond) and median (black horizontal line) of $\Delta r$ is also plotted.

As described in Section 3.2, the arc-tangent loss function is expected to have a superior performance over the linear loss function in the presence of outliers. In addition to the localization error, the cumulative probability distribution of the predicted $\Delta r$ is shown for the linear loss function.

Figure 3 shows the localization with linear loss function in the absence of disruptions (no barrier, no noise, and all receivers functioning) in case of random position initialization. The observed absolute distance between the true location and the estimated location is $\Delta r=0.0126 \mathrm{~m}$.

Section 5.2 presents the result with only one single disruption. Section 5.3 presents the results with two disruptions present simultaneously, because, in reality, the combination of two or more disruptions is more likely.

\subsection{Single Disruptions}

Figure 5a depicts the localization error in the presence of barriers for different cases of barrier orientation (Figure 4) estimated both for linear and arc-tangent loss functions. Figure $5 b$ shows the cumulative distribution of predicted $\Delta r$ plotted for both the loss functions. In the presence of a barrier, the direct line-of-sight paths between the sender and some of the receivers are obstructed. This induces outliers in the TDOAs and $\Delta d_{i j}$, and subsequently errors in the location estimated using Equation (10). The results in Figure 5a show that the arc-tangent loss function, which is more robust against outliers, results in smaller localization errors $(\Delta r)$ than the linear loss function.

Figure 6a depicts the localization error in the presence of noise, using box plots. Figure $6 \mathrm{~b}$ shows the cumulative distribution of predicted $\Delta r$ for both the loss functions. Introducing noise into the system introduces significant localization errors, which increases up to $6 \mathrm{~m}$ as the level of noise increases (Figure 6a). The error increases as the wrong peak is extracted from the cross-correlation function as described in Section 3.1. This leads to erroneous localization estimates as unreliable TOAs and hence TDOAs are used in Equation (10), resulting in outliers. Arc-tangent loss function being robust to outliers performs well in the presence of noise (Figure 6a). 


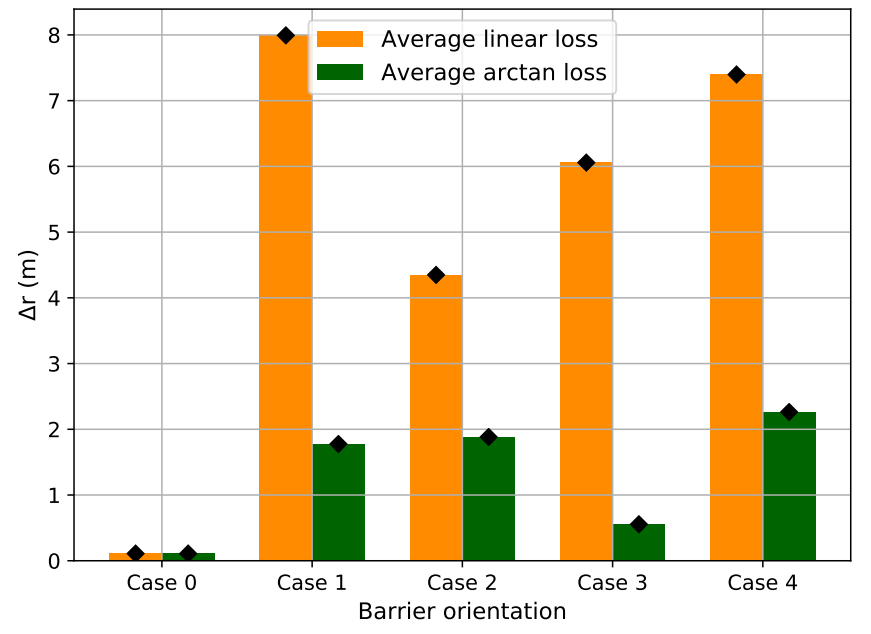

(a)
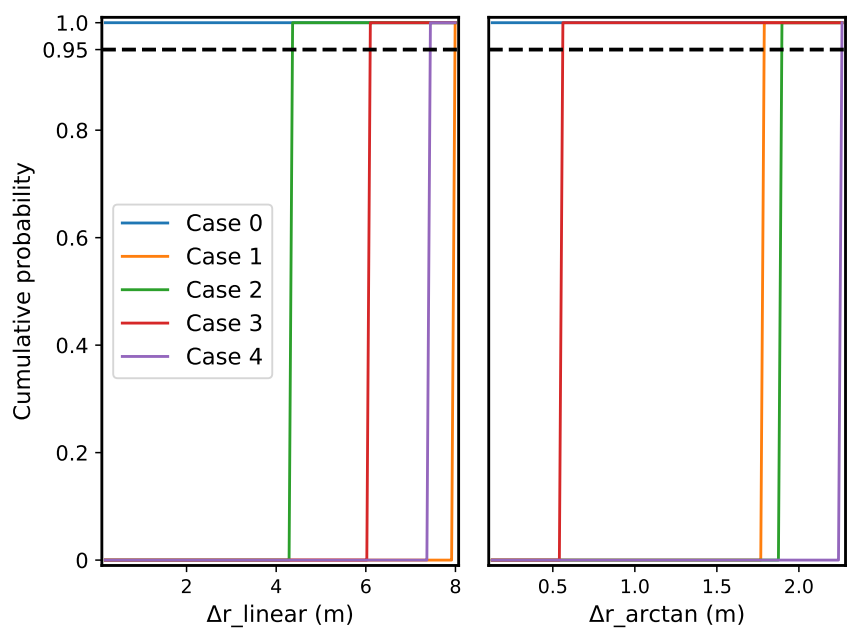

(b)

Figure 5. (a) Localization error for the barrier as disruption (with different barrier orientation as shown in Figure 4). For a static source, the location estimation is the same for a fixed barrier, thus generating the same localization error for each instance for a given case. (b) Cumulative distribution of $\Delta r$ for the linear and arc-tangent loss functions. As the location estimation is the same for a given barrier case, the cumulative function looks like a step function.

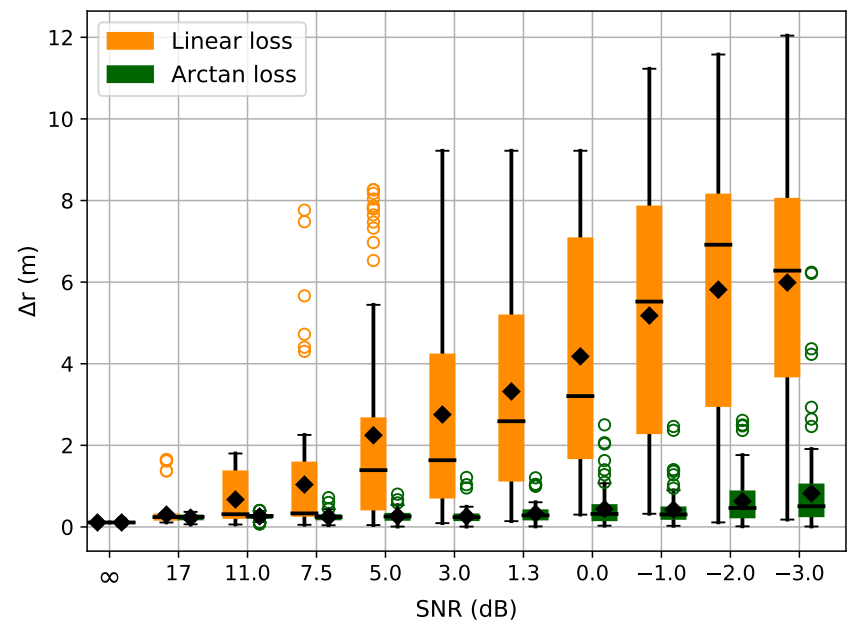

(a)
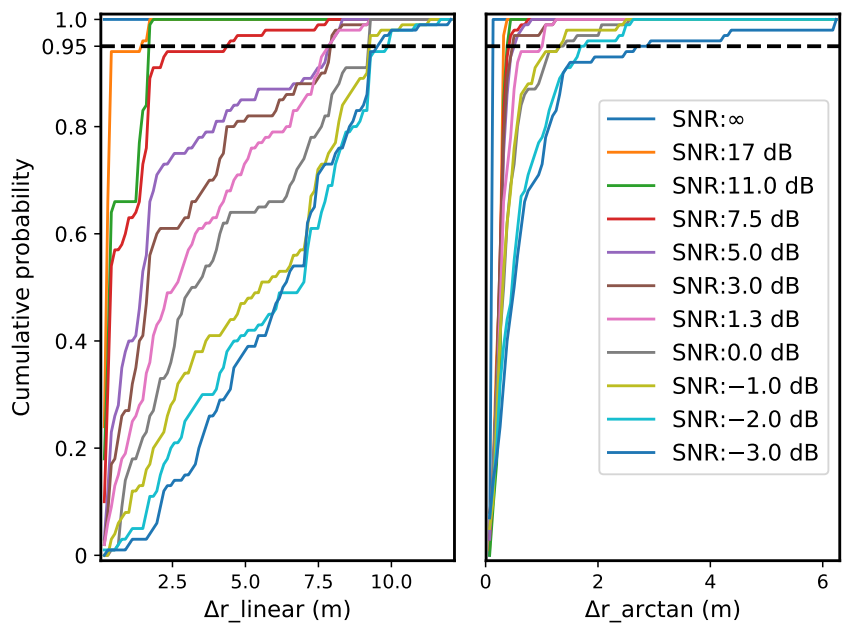

(b)

Figure 6. (a) Localization error with noise as disruption. The signal-to-noise ratio is given for each increasing noise level. (b) Cumulative distribution of $\Delta r$ for the linear and arc-tangent loss functions. As the noise in the system increases, the localization error shows a higher variance. The variance is comparatively less for the arc-tangent loss function.

Figure 7 shows localization error for receiver malfunction and cumulative distribution of predicted $\Delta r$ plotted for both the loss functions. For receiver malfunction, reduced localization accuracy is visible when the number of active receivers reduces to perform the localization (Figure 7a). For this disruption, both arc-tangent and linear loss functions show comparable performance (Figure 7a). In this case, they have to cope with the system malfunction, which does not introduce any outliers, but rather reduces the number of TDOA values $d_{\mathrm{ij}}$ available to solve the localization problem (Equation (10)). 


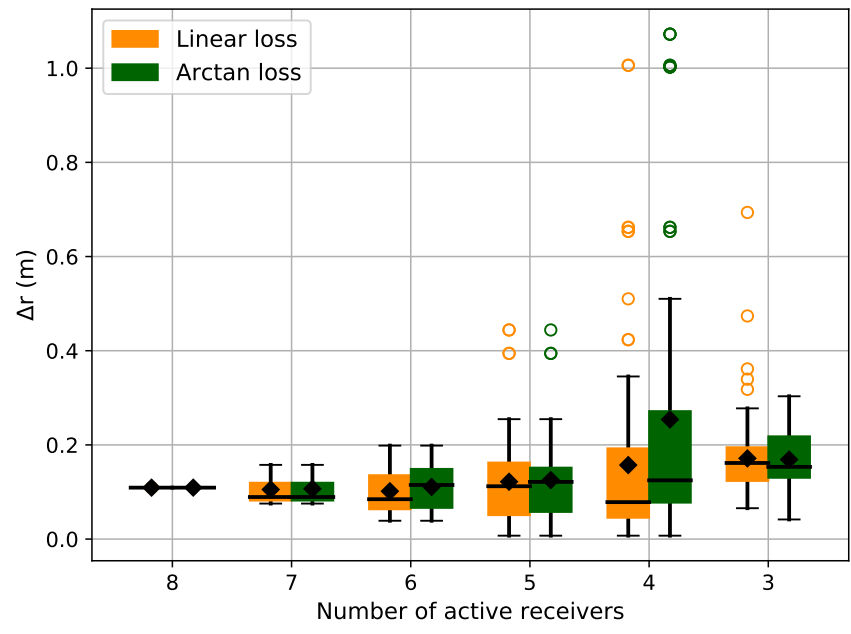

(a)
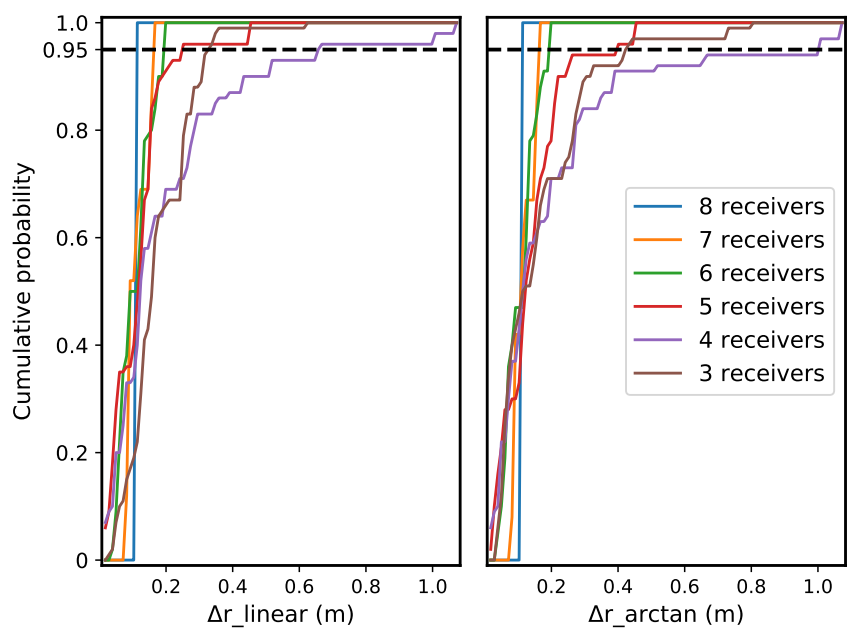

(b)

Figure 7. (a) Localization error for receiver malfunction as disruption for a system with 8 receivers. The different cases are generated by reducing the number of active receivers randomly from 8 to 2. (b) Cumulative distribution of $\Delta r$ for the linear and arc-tangent loss functions. The cumulative distribution is almost similar for both the loss functions.

It is also observed that even if the number of available receivers is only three, the observed $\Delta r$ is small if the initialization of the position is close to the real position. The reason is that the objective function in Equation (5) is not concave and a good initialization ensures that it converges to a local minimum that is close to the global minimum. Another observation is the sudden increase in $\Delta r$ for four receivers (Figure $7 \mathrm{a}$ ), which is due to the maximum number of random combinations of receivers possible, i.e., $\left(\begin{array}{l}8 \\ 4\end{array}\right)$.

The consolidated comparison between arc-tangent and linear loss is presented in Table 1. For barrier, the mean values of case 2 barrier are selected. For noise, the mean values at $\mathrm{SNR}=5.0 \mathrm{~dB}$ are extracted. And for receiver malfunction, the mean values at 5 active receivers are used. These values are selected to have a representative amount of each disruption.

Table 1. Comparison of localization error between arc-tangent and linear loss functions for single disruptions. From all the plots one single value is extracted for comparison. The extracted values are: Case 2 barrier, $\mathrm{SNR}=5.0 \mathrm{~dB}$ and 5 active receivers.

\begin{tabular}{cccc}
\hline Loss Function & Barrier (Figure 5) & Noise (Figure 6) & Receiver Malfunction (Figure 7) \\
\hline Linear & $4.2 \mathrm{~m}$ & $2.2 \mathrm{~m}$ & $0.12 \mathrm{~m}$ \\
Arc-tangent & $1.9 \mathrm{~m}$ & $0.2 \mathrm{~m}$ & $0.12 \mathrm{~m}$ \\
\hline
\end{tabular}

\subsection{Multiple Disruptions}

In this section, we combined two disruptions, which is a more realistic scenario, together and then evaluated the system's performance. In all the evaluations, one disruption is kept constant and the other disruption is changed. Although there could be several more sets of two disruptions, we evaluated only three combinations in the present study. The first combination is constant barrier plus increasing noise. The second combination is constant noise and reducing the number of receivers. And the third combination is a constant number of available receivers and increasing noise. All the constant values are chosen at random to have a representative value of the disruption.

Figure 8a shows the localization error with barrier and increasing noise. The barrier is fixed (Case 2 of Figure 4) and noise is increasing as in Figure 6. Figure 8 b shows the cumulative distribution of predicted $\Delta r$ for both the loss functions. When comparing Figures $6 \mathrm{a}$ and $8 \mathrm{a}$, it becomes evident that even a small amount of noise has a strong negative influence on the localization accuracy if the localization is already disrupted with 
a barrier. In Figure 8a, the initial performance gap between the two loss functions is due to the superiority of the arc-tangent in the presence of a barrier. Further observation is also in agreement with what is observed in Figure 6a, i.e., that the arc-tangent has a better performance when the SNR decreases.

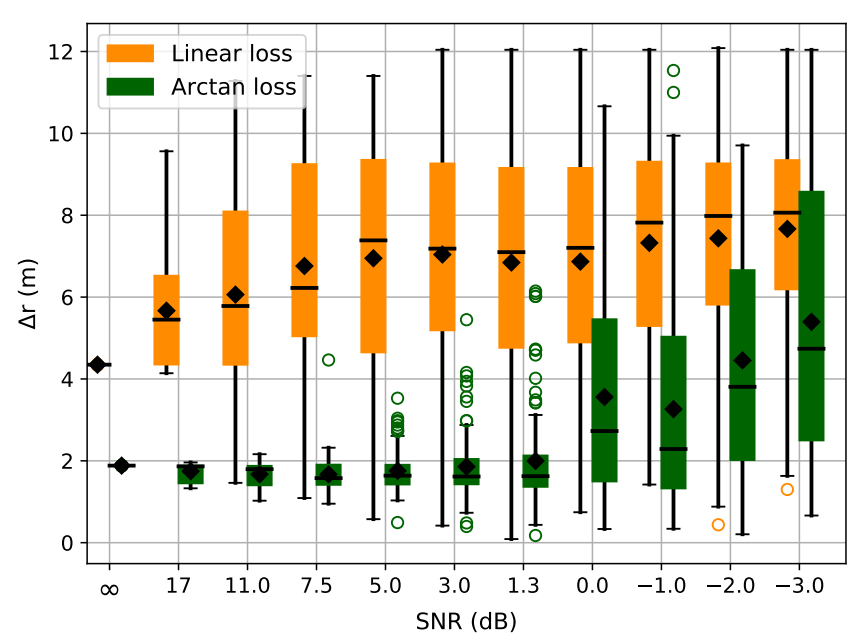

(a)
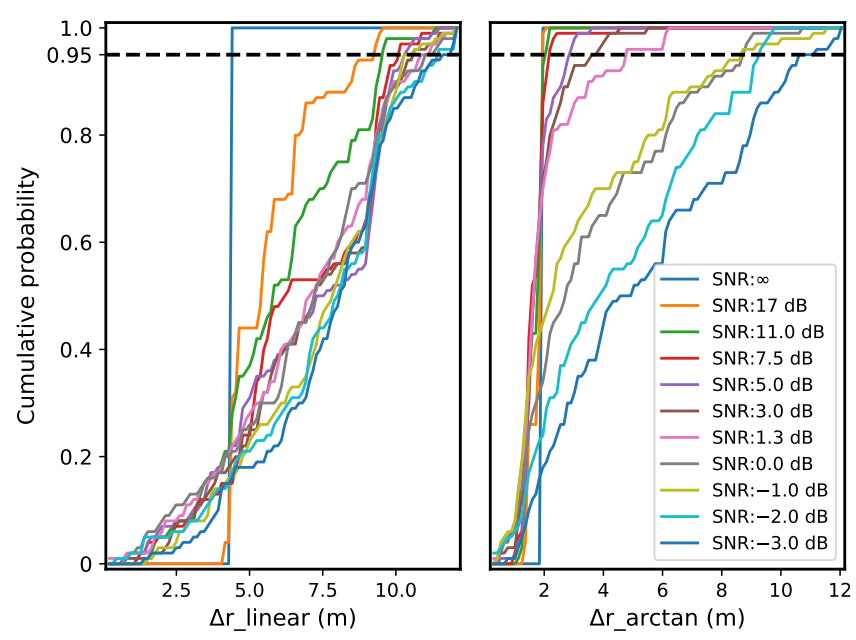

(b)

Figure 8. (a) Localization error with barriers and increasing noise. Barrier orientation is as in Case 2 of Figure 4 . The white noise of zero means is varied with increasing standard deviation from 0 to 1 in steps of 0.1. (b) Cumulative distribution of $\Delta r$ for the linear and arc-tangent loss functions.

Figure 9a shows the effect of decreasing the receiver's numbers for a given noise level. It is observed that the localization accuracy is further reduced if, in addition to the reduced number of active receivers, the noise is also present, as deduced when comparing Figures 7a and 9a. It can be seen in Figure 9a that the arc-tangent shows superior performance compared to the linear loss function. The initial performance gap between arc-tangent and linear loss is due to the presence of a constant noise of mean $\mu=0$ and standard deviation $\sigma=0.4$ corresponding to $\mathrm{SNR}=5.0 \mathrm{~dB}$. In this case, when reducing the number of active receivers, the arc-tangent keeps performing better. In the present case, the dominating performance of the arc-tangent is due to the hybrid nature of the disruptions, thus concluding that the arc-tangent shows superior performance for realistic disruption scenarios.

Figure 10 shows the effect of increasing noise when some receivers are already malfunctioning. When comparing the localization error with 5 receivers selected at random, the effect of noise on localization errors (Figure 10a) is found to be similar to the case of all receivers working (see Figure 6a). This is because the constant malfunctioning of three inactive receivers only causes minor localization errors. The absence of initial performancegap between the two loss functions in comparison to Figures $8 \mathrm{a}$ and $9 \mathrm{a}$ is because both arc-tangent and linear loss functions show a comparable performance for pure receiver malfunction (Figure 7a). As the noise is introduced in the system, the superiority of the arc-tangent loss function can be seen. 


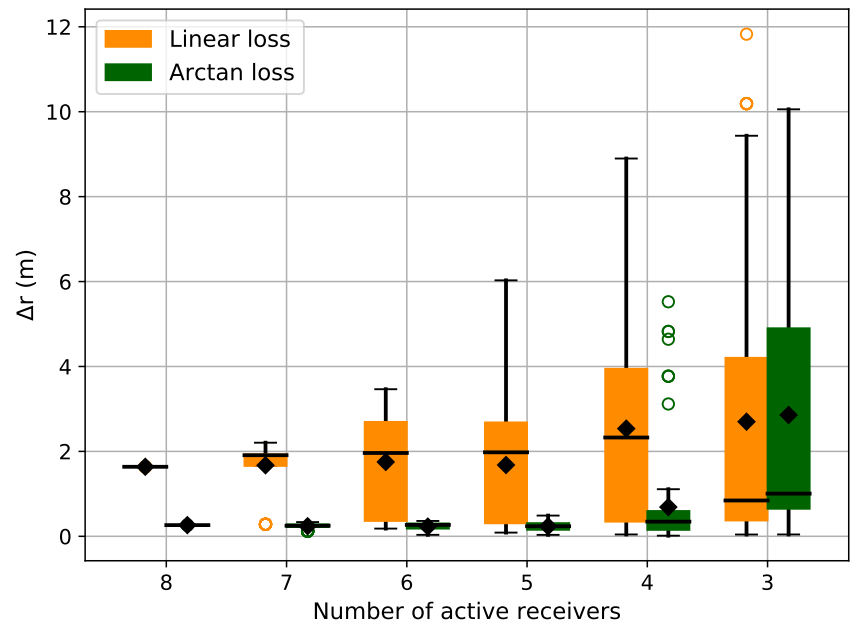

(a)
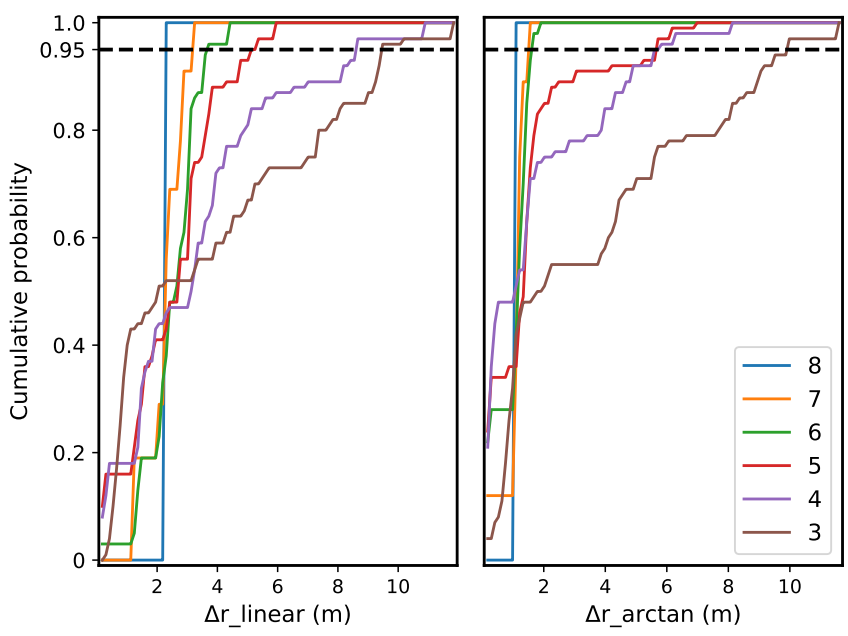

(b)

Figure 9. (a) Localization error plots with constant noise and reducing the number of active receivers. Throughout the simulation, white noise of $\mu=0$ and $\sigma=0.4$ corresponding to the $\mathrm{SNR}=5.0 \mathrm{~dB}$ is present. The numbers of active receivers are decreased from 8 to 2. (b) Cumulative distribution of $\Delta r$ for the linear and arc-tangent loss functions.

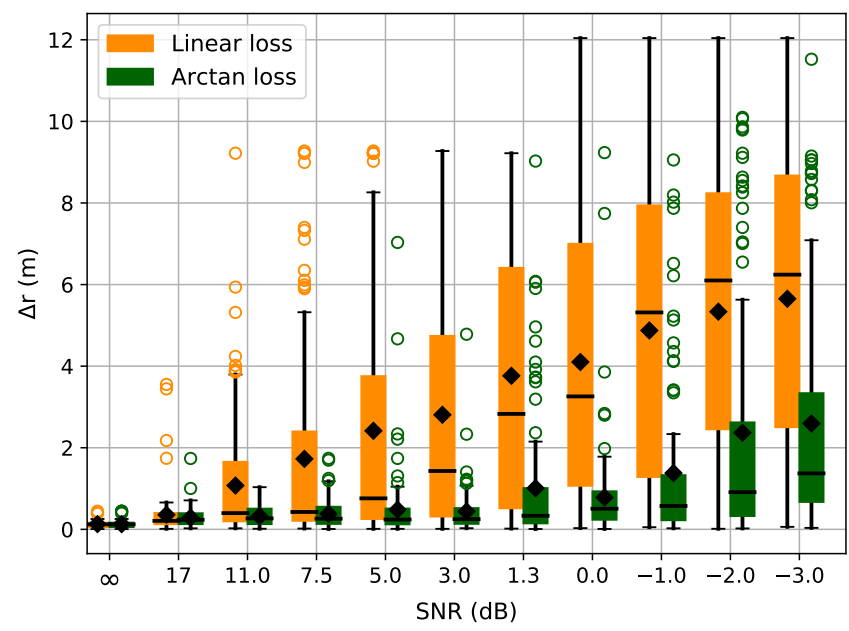

(a)
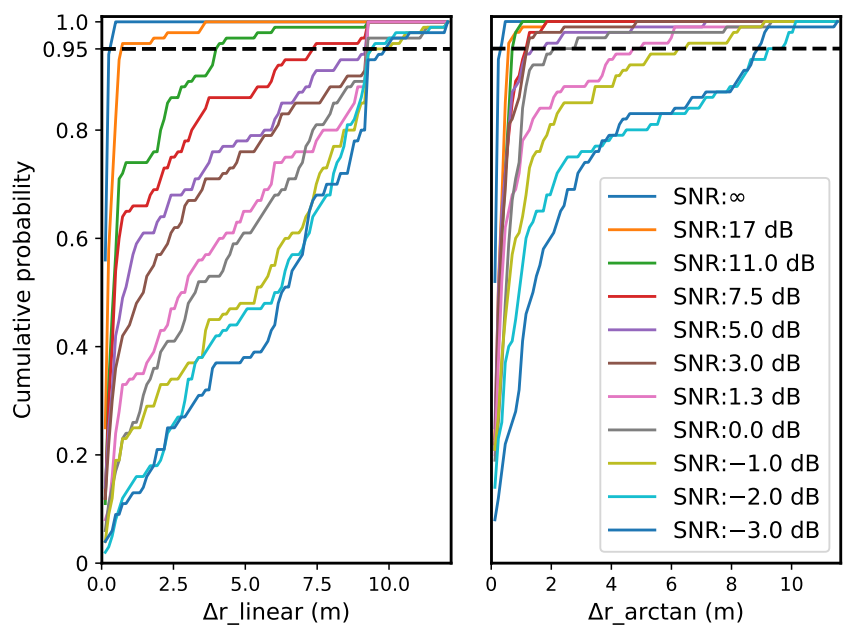

(b)

Figure 10. (a) Localization error with 5 active randomly selected receivers and increasing noise. (b) Cumulative distribution of $\Delta r$ for the linear and arc-tangent loss functionsn.

The consolidated comparison between arc-tangent and linear loss is presented in Table 2. For barrier plus noise, the mean values of barrier Case 2 and an SNR $=5.0 \mathrm{~dB}$ were selected. For noise plus receiver malfunction, the mean values at $\mathrm{SNR}=5.0 \mathrm{~dB}$ with 5 active receivers were extracted. And for receiver malfunction plus noise, the mean values at 5 active receivers with $\mathrm{SNR}=5.0 \mathrm{~dB}$ were used. The last two values represent the same scenario and thus they also have comparable localization errors. The difference appears because of different simulation runs. These values are selected to have a representative amount of each disruption. 
Table 2. Top-level comparison of localization error between arc-tangent and linear loss functions for multiple disruptions. From all the plots one single value is extracted for comparison. The extracted values are: Case 2 barrier, $\mathrm{SNR}=5.0 \mathrm{~dB}$ and 5 active receivers.

\begin{tabular}{cccc}
\hline Loss Function & Barrier + Noise (Figure 8) & $\begin{array}{c}\text { Noise + Receiver Malfunction } \\
\text { (Figure 9) }\end{array}$ & $\begin{array}{c}\text { Receiver Malfunction + Noise } \\
\text { (Figure 10) }\end{array}$ \\
\hline Linear & $6.4 \mathrm{~m}$ & $1.9 \mathrm{~m}$ & $2.1 \mathrm{~m}$ \\
Arc-tangent & $1.8 \mathrm{~m}$ & $0.2 \mathrm{~m}$ & $0.2 \mathrm{~m}$ \\
\hline
\end{tabular}

\subsection{Time-Dependent Resilience Curves for Linear Loss Function}

In this section, the time-dependent resilience curves [4] are studied. The timedependent resilience curve generation is based on the following modeling assumptions:

1. Consecutive chirps according to Equation (2) are spaced by $50 \mathrm{~ms}$.

2. The computations are conducted with no delay between chirps.

3. Disruptions appear and disappear instantaneously, which is a simplification but very powerful for comparison of pure resilience response and recovery behavior. The duration of the disruptions is $5 \mathrm{~s}$.

4. A uniform backward-looking moving average as in Equation (11) is plotted for $N_{\mathrm{w}}=30$ instances in each case corresponding to a window of $1.5 \mathrm{~s}$.

5. The first sender position initialization is random. Then, initialization is carried out using the estimation of the previous time step.

Figure $11 \mathrm{a}$ assesses the time-dependent resilience behavior for a barrier of Case 2 shown in Figure 4 present for $T=5 \mathrm{~s}$ starting at $t_{1}=5 \mathrm{~s}$ and ending at $t_{2}=10 \mathrm{~s}$. Figure $11 \mathrm{~b}$ adds noise with $\mu=0, \sigma=0.4$ which corresponds to SNR $=5 \mathrm{~dB}$. Figure 11c shows the effect of switching off, at random, four receivers. In both cases, the disruption is introduced and removed at the same times as in Figure 9a.

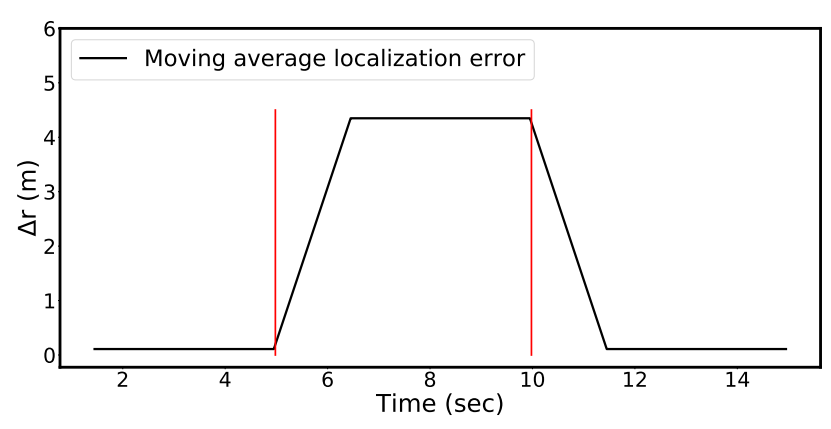

(a)

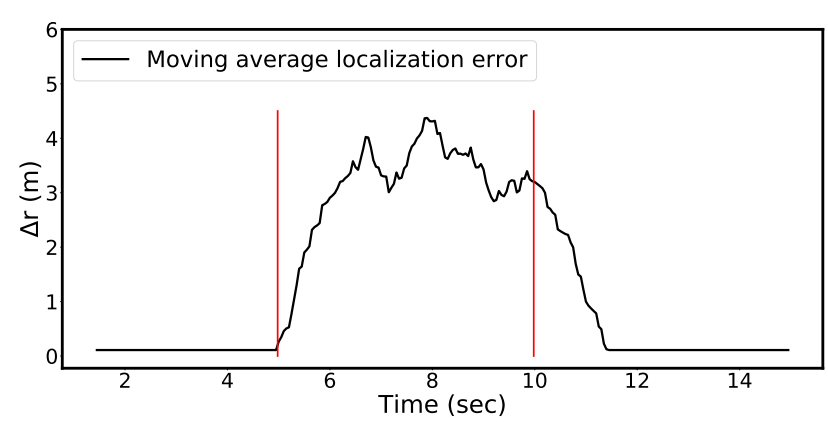

(b)

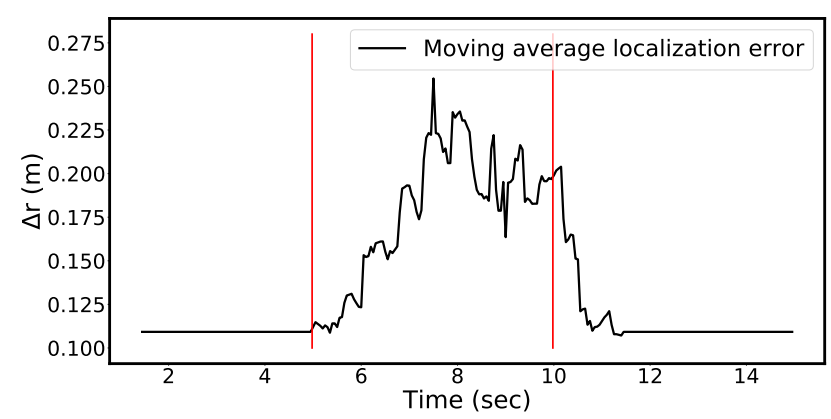

(c)

Figure 11. Time-dependent resilience curves with (a) barrier as disruption, (b) noise as disruption, and (c) receiver malfunction as disruption. The red lines mark start time $t_{1}=5 \mathrm{~s}$ and end time $t_{1}=10 \mathrm{~s}$ of the disruptions detailed in the main text. 
The behavior seen in Figure 11a,b is as expected when inspecting Figures $5 \mathrm{a}$ and $6 \mathrm{a}$ and taking the smoothing described under assumption 4into account. The time-dependent resilience response curve in Figure 11c shows that the smoothing has a rather small effect due to the large deviation in case of the random disruption present (Figure 7a). The latter type of disruption is physically not very likely when compared to the single loss of all but four receivers. However, all cases show benign degradation (e.g., there are no oscillations) and that the implemented algorithm recovers fast after all types of disruptions.

\subsection{Resilience Quantification of Indoor Localization System}

To apply "Rsl" (Equation (15)) to the simulations, the performance values $r_{\mathrm{j}}, t_{\mathrm{j}}$, performance tolerance $R_{0}$ and time tolerance $T_{0}$ are needed. Sections 5.2 and 5.3 present the simulation results of the performance during a disruption, i.e., the values $r_{3 \mathrm{j}}, r_{4 \mathrm{j}}$. Additionally, Section 5.4 provides the information about the disruption time, i.e., $t_{2 \mathrm{j}}, t_{5 \mathrm{j}}$ and $t_{6 \mathrm{j}}$.

The simulations are distinct between two different objective functions for the nonlinear least-square optimization: first, a linear loss function, which is equal to the well-known linear least-square fit; and secondly, an arc-tangent loss function, where the arcus-tangent of a quadratic sum is minimized. In Figure 12, the red values quantify cost functions in the linear case, the green in arc-tangent fit. The values are dimensionless, because they are based on the performance tolerance level $R_{0}$ and the time tolerance duration $T_{0}$, and they are always positive.

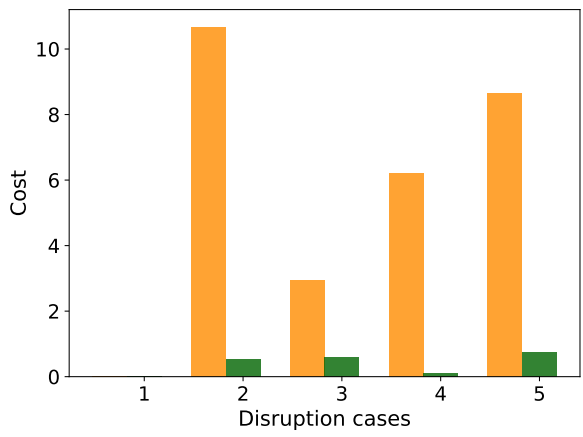

(a)

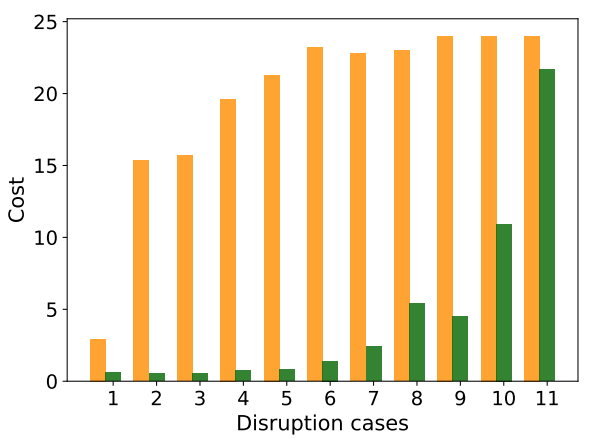

(d)

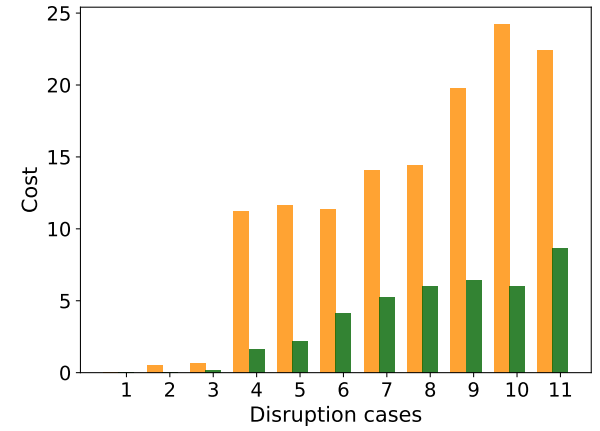

(b)

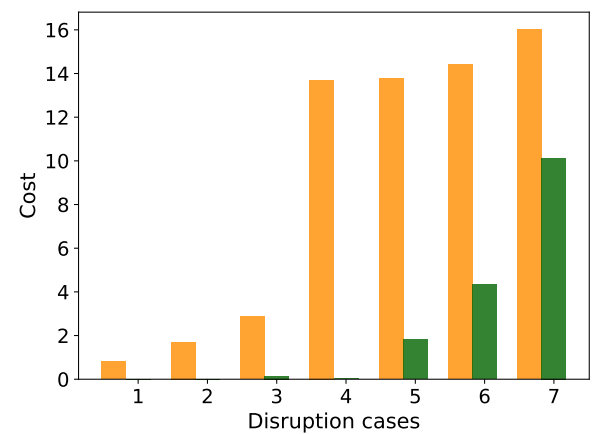

(e)

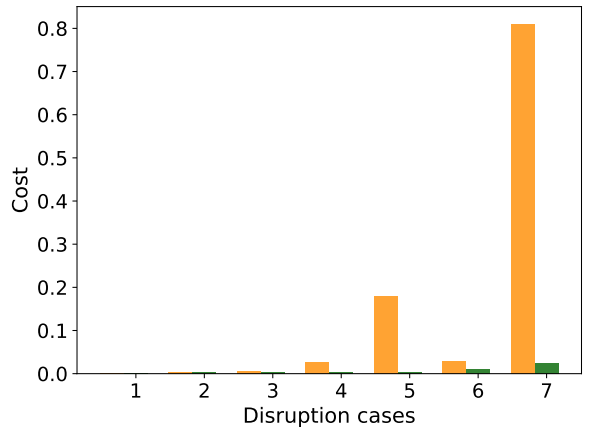

(c)

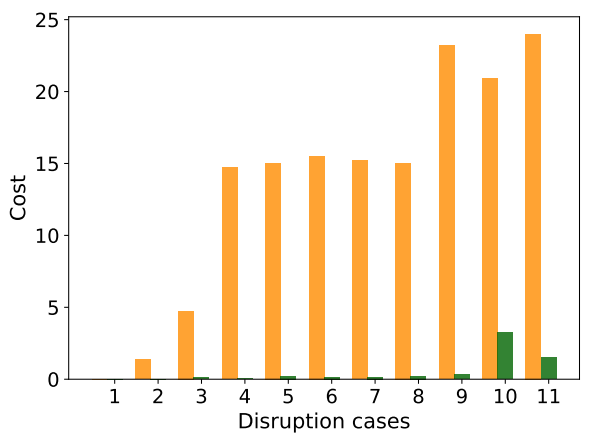

(f)

Figure 12. Cost function quantification and comparison between linear and arc-tangent loss function. Red color refers to the linear loss and green color refers to the arc-tangent loss. The diagrams are directly related to the simulation cases (a) barrier disruption, (b) noise disruption, (c) receiver malfunction, (d) barrier (case 2 of Figure 4) plus increasing noise, (e) fixed noise $(\mathrm{SNR}=5 \mathrm{~dB})$ plus receiver malfunction (reducing number of receivers), $(\mathbf{f})$ receiver malfunction (three receivers are malfunctioning) plus increasing noise.

Figure 12 shows that the cost functions in the case of arc-tangent fits are always smaller than in the linear case. So, it is clear that the arc-tangent fit is an improvement. Quantifying this in resilience calculus, we note that the cost functions are a part of resilience. Generally, 
Equation (15) is the e-function of a scalar product of $\vec{a}$ and $\vec{b}$ (Equation (12)). Since all components are positive, then: $0 \leq R s l \leq 1$, where 0 means complete destruction of a system. In the case of small $a$-values, the disruptions are rare, and $R s l$ is close to 1 . If the disruptions are frequent, the scalar product grows, and $R s l$ decreases. It is a sum, i.e., one severe disruption can cause a low resilience, even if all other components are low. In such a case, the system is not well suitable for this kind of disruption, which can be caused by high $a$ (happens often) or by high $b$ (the system robustness against the disruption is bad) or both.

The probabilities of various disruptions in an indoor localization system are

1. Barrier: $a_{\mathrm{B}}=0.0052$ in every case.

2. Noise: Sum of $a_{\mathrm{N}}=0.02$ decreasing level.

3. Receiver malfunction: Sum of $a_{\mathrm{RM}}=0.046$ decreasing level.

Combining the probabilities with the cost function result in resilience quantities for the simulated system that is displayed in the Table 3:

Table 3. Resilience quantification of the simulated indoor acoustic localization system for both arc-tangent and linear loss functions.

\begin{tabular}{cc}
\hline Loss Function & Resilience $($ Rsl $)$ \\
\hline Linear & 0.634 \\
Arc-tangent & 0.936 \\
\hline
\end{tabular}

The results in Table 3 shows that the arc-tangent loss function is more resilient than the linear loss function.

\section{Experimental Results}

In this section, we experimentally verify whether the improvements observed when using the simulation environment can also be observed when using measured data. Previously, we found that the arc-tangent loss function provides more accurate location estimates than the linear loss function. Here, we verify whether this is true also for the data collected through the experiments. Although this section uses experimental data for the verification, it does not attempt to validate the simulations. Instead, we try to state that this simulation framework can be used to test acoustic indoor localization systems with a certain credibility.

The experimental site is shown in Figure 13a. The room is $29.1 \mathrm{~m}$ long and $29.7 \mathrm{~m}$ wide. For the first experiment, we installed 16 receivers in the room. The room dimension and corresponding arrangement of receivers are depicted in Figure 13a. For the experimental results, we repeated the static experiment for each source position and performed the localization using both arc-tangent and linear loss functions. For each source location, we ran the evaluation 500 times and plotted the results using the bar plots in Figure 13b.

As can be seen in Figure 13b, arc-tangent performs better than the linear loss function for different source locations. The reason being, arc-tangent is better in handling outliers (Section 3) i.e., the arc-tangent loss function is less sensitive to wrongly extracted TOAs than the linear loss function. 


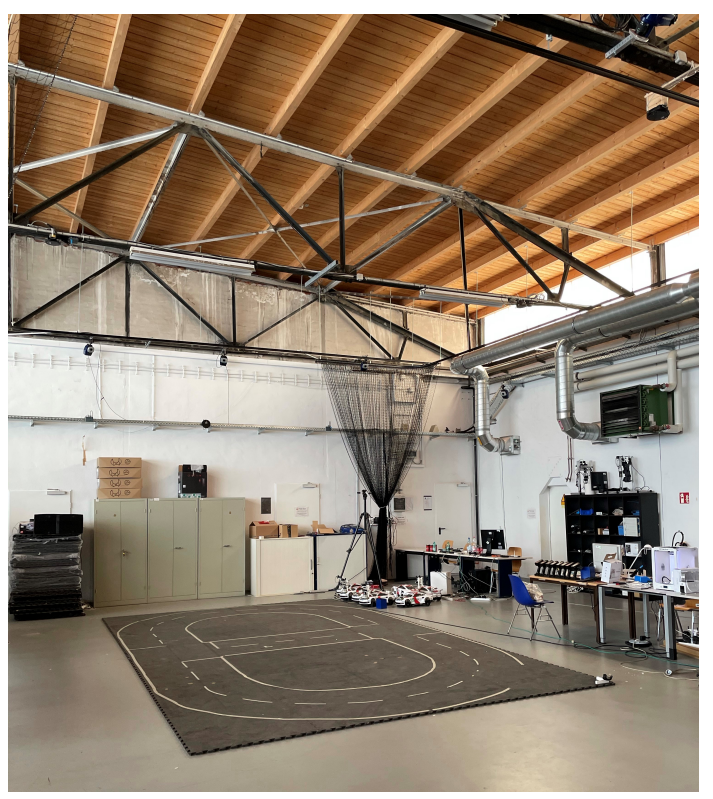

(a)

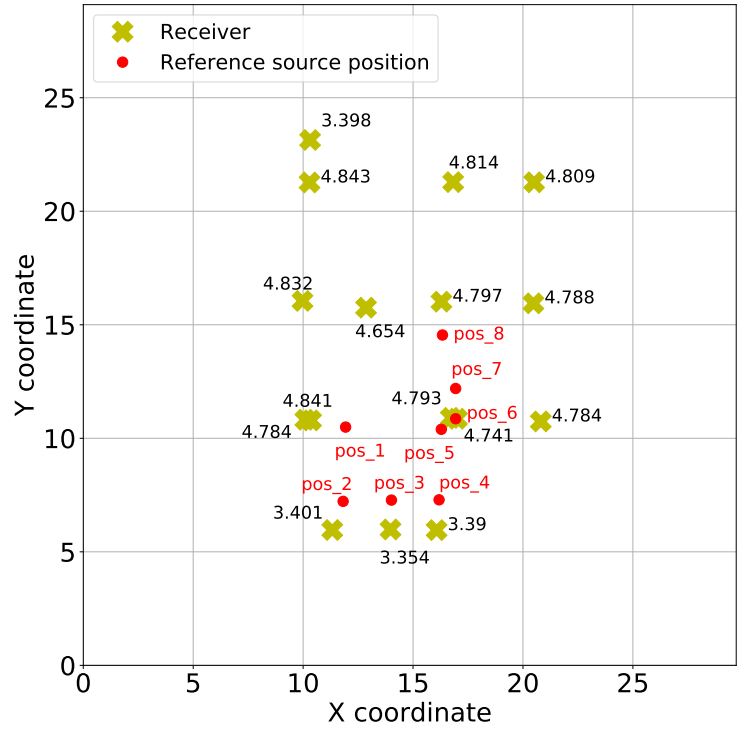

(b)

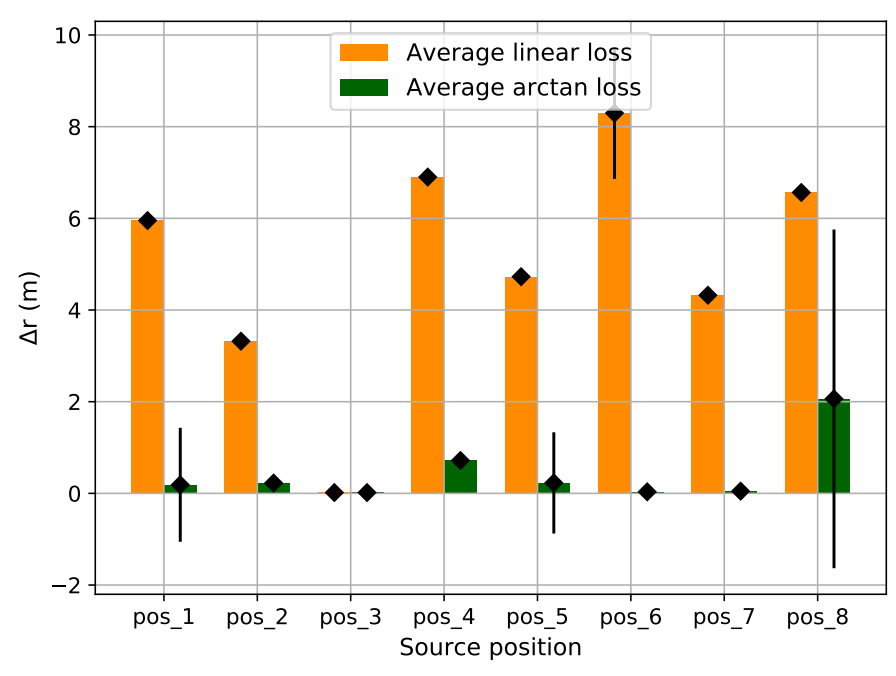

(c)

Figure 13. (a) Physical experimental site. Room dimensions are $29.1 \mathrm{~m} \times 29.7 \mathrm{~m}$. (b) Yellow crosses are the receiver with corresponding height written next to them. All the red dots represent the reference source positions on the ground. (c) Localization error for different source locations and loss functions.

For the next experiment, we used one specific source position (pos 3 ) and selected 8 receivers out of 16 with the highest SNR. These receivers are shown in Figure 14a. In the experiment, we reduced the number of receivers from 8 to 3 and compared the localization accuracy using both arc-tangent and linear loss functions. For each case (number of receivers), we repeated the evaluation 500 times, where the active receivers were selected at random from the set of 8 receivers. The results are shown in Figure 14b. Figure 14c is the snapshot of Figure $14 \mathrm{~b}$ to better visualize the performance of arc-tangent and linear when the number of receivers is more than 4 . 


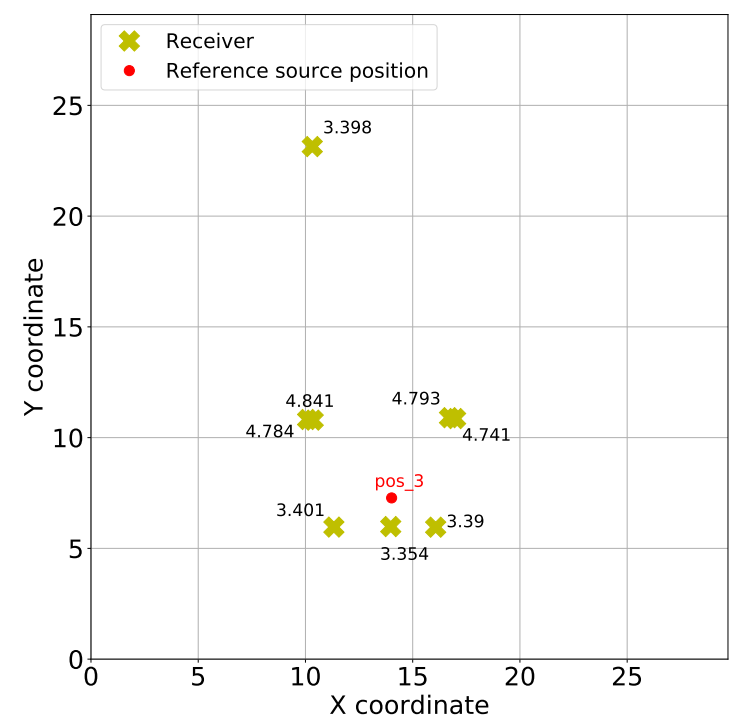

(a)

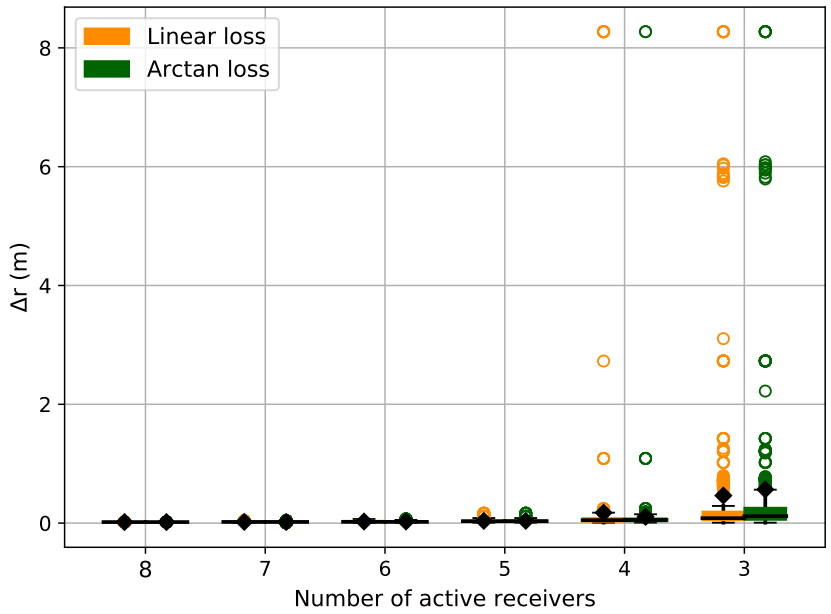

(b)

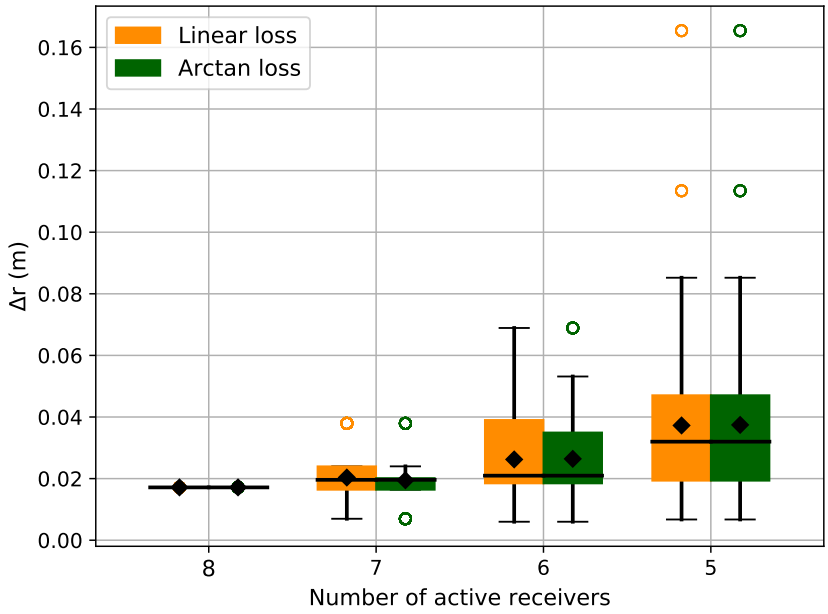

(c)

Figure 14. (a) Experimental setup: Yellow crosses are the receiver with corresponding heights written next to them. Only 8 receivers are selected with best SNR. The red dots represent the reference source position on the ground. (b) Localization error for different number of active receivers and loss functions, and (c) Snapshot of (b) for more than 4 receivers.

In Figure 14b, we can see that for a given source, when the number of available receivers is reduced from 8 to 3 , both arc-tangent and linear loss functions have the same performance. In this case, it is the receiver malfunction that is acting as disruption. Reducing the number of available receivers does not introduce any outliers but reduces the number of available variables to localize the source accurately. And since we used only those receivers with very high SNR, we assumed that the TOAs are extracted accurately, which eliminates the factor of arc-tangent performing better than the linear loss function because of wrongly extracted TOAs.

\section{Conclusions}

An end-to-end simulation framework was developed for a sample acoustic indoor localization system, and its usefulness was demonstrated by evaluating and analyzing the robustness and resilience of the system in the presence of disruptions. The framework was used to simulate and assess the behavior of the sample system in the presence of individual disruptions and the combination of disruptions, which allowed us to investigate inter-dependencies of disruptions systematically. The approach enables modeling the 
expected effects of disruptions present only for a limited time, particularly the localization accuracy loss as soon as the disruption is present (time-dependent response to disruption) and the accuracy gain after it is removed (recovery bounce-back). Such time-dependent assessments are essential for resilience analysis in operational contexts. The resilience of the sample system was also quantified.

It was found that the arc-tangent loss function can drastically improve the system performance in the presence of single disruptions, except in the case of pure receiver malfunction. However, when combining the disruptions (hybrid disruptions) for more realistic disruption scenarios, the performance of the arc-tangent loss function always dominates the performance of the linear loss function. It was also shown that the system is highly resilient to disruptions when using the arc-tangent loss function.

Future work may consist of an evaluation of this scheme for more (acoustically) complex room geometries and multiple acoustic sources including complex situations like multi-path effect and multiple occlusions. Additional disruptions could also be introduced. It is also of great interest to extend this method to simulate moving sources and timedependent disruptions appearing in time and space to generate true time-dependent behavior, in particular, to generate resilience curves.

Author Contributions: Conceptualization, A.K.J., I.H., and E.A.P.H.; data curation, A.K.J.; formal analysis, A.K.J. and P.G.; funding acquisition, D.J.S., I.H., F.H., C.S., H.S., P.G., and S.J.R.; investigation, A.K.J.; methodology, A.K.J. and H.S.; project administration, A.K.J., I.H., F.H., C.S., H.S., P.G., and S.J.R.; resources, A.K.J., F.H., C.S., H.S., D.J.S., S.J.R., and G.F.; software, A.K.J.; supervision, E.A.P.H., I.H., and P.G.; validation, A.K.J., H.S., and G.F.; visualization, A.K.J. and G.F.; writing-original draft preparation, A.K.J., D.J.S., and H.S.; writing—review and editing, A.K.J., D.J.S., I.H., G.F., F.H., H.S., E.A.P.H., and P.G. All authors have read and agreed to the published version of the manuscript.

Funding: All authors but E. H. acknowledge partial support of this work through a grant of the Sustainability Center Freiburg for the pilot research project MERLIN, funded by the German Federal State of Baden-Württemberg in the Framework of the Fraunhofer-Gesellschaft, and also the German Ministry of Education and Research (BMBF) under the grant FKZ 16ME0023K ("Intelligentes Sensorsystem zur autonomen Überwachung von Produktionsanlagen in der Industrie 4.0-ISA4.0").

Institutional Review Board Statement: Not applicable.

Informed Consent Statement: Not applicable.

Data Availability Statement: The data presented in this study are available on request from the corresponding author.

Conflicts of Interest: The authors declare no conflict of interest.

\begin{tabular}{|c|c|}
\hline \multicolumn{2}{|c|}{ Abbreviations } \\
\hline \multicolumn{2}{|c|}{ The following abbreviations are used in this manuscript: } \\
\hline ASSIST & acoustic self-calibrating system for indoor smartphone tracking \\
\hline CPS & cross power spectrum \\
\hline RFID & radio frequency identification \\
\hline RIR & room impulse response \\
\hline SNR & signal-to-noise ratio \\
\hline TDOA & time difference of arrival \\
\hline TOA & time of arrival \\
\hline
\end{tabular}

\section{References}

1. Häring, I. Risk Analysis and Management: Engineering Resilience; Springer: Singapore, 2015. [CrossRef]

2. Häring, I.; Scharte, B.; Hiermaier, S. Towards a novel and applicable approach for resilience engineering. In Proceedings of the 6th International Disaster and Risk Conference (IDRC), Davos, Switzerland, 28 August-1 September 2016; pp. $272-276$.

3. Häring, I.; Scharte, B.; Stolz, A.; Leismann, T.; Hiermaier, S. Resilience engineering and quantification for sustainable systems development and assessment: Socio-technical systems and critical infrastructure. IRGC Resour. Guide Resil. $2016,1,9$. 
4. Häring, I.; Ebenhöch, S.; Stolz, A. Quantifying resilience for resilience engineering of socio technical systems. Eur. J. Secur. Res. 2016, 1, 21-58. [CrossRef]

5. Liu, M.; Cheng, L.; Qian, K.; Wang, J.; Wang, J.; Liu, Y. Indoor acoustic localization: A survey. Hum.-Centric Comput. Inf. Sci. 2020, 10, 2. [CrossRef]

6. Huang, Y.; Benesty, J.; Chen, J. Time Delay Estimation and Source Localization. In Springer Handbook of Speech Processing; Benesty, J., Sondhi, M.M., Huang, Y.A., Eds.; Springer: Berlin/Heidelberg, Germany, 2008; Chapter 51.

7. Comuniello, A.; Moschitta, A.; Angelis, A.D. Ultrasound TDoA Positioning Using the Best Linear Unbiased Estimator and Efficient Anchor Placement. IEEE Trans. Instrum. Meas. 2020, 69, 2477-2486. [CrossRef]

8. Peng, C.; Shen, G.; Zhang, Y.; Li, Y.; Tan, K. BeepBeep: A high accuracy acoustic ranging system using COTS mobile devices. In Proceedings of the 5th International Conference on Embedded Networked Sensor Systems, SenSys'07, Sydney, Australia, 6-9 November 2007; pp. 1-14. [CrossRef]

9. Hoflinger, F.; Hoppe, J.; Zhang, R.; Ens, A.; Reindl, L.; Wendeberg, J.; Schindelhauer, C. Acoustic indoor-localization system for smart phones. In Proceedings of the 2014 IEEE 11th International Multi-Conference on Systems, Signals \& Devices (SSD14), Barcelona, Spain, 11-14 February 2014. [CrossRef]

10. Langlois, C.; Tiku, S.; Pasricha, S. Indoor Localization with Smartphones: Harnessing the Sensor Suite in Your Pocket. IEEE Consum. Electron. Mag. 2017, 6, 70-80. [CrossRef]

11. Ens, A.; Höflinger, F.; Zhang, R.; Hoppe, J.; Bannoura, A.; Reindl, L.; Wendeberg, J.; Buhrer, M.; Schindelhauer, C. Acoustic self-calibrating system for indoor smartphone tracking (ASSIST). Int. J. Navig. Obs. 2012, 2015, 1-9. [CrossRef]

12. Sapumohotti, C.; Alias, M.Y.; Tan, S.W. WILOCSIM: Simulation Testbed for WLAN Location Fingerprinting Systems. Prog. Electromagn. Res. B 2013, 46,1-22. [CrossRef]

13. Alhammadi, A.; Hashim, F.; Rasid, M.F.A.; Alraih, S.; Aquhali, A. Simulation and Experimental Analysis of Indoor Localization Systems. In Proceedings of the 2019 International Seminar on Research of Information Technology and Intelligent Systems (ISRITI),Yogyakarta, Indonesia, 5-6 December 2019. [CrossRef]

14. Allen, J.B.; Berkley, D.A. Image method for efficiently simulating small-room acoustics. J. Acoust. Soc. Am. 1979, 65, 943-950. [CrossRef]

15. Kirszenstein, J. An image source computer model for room acoustics analysis and electroacoustic simulation. Appl. Acoust. 1984, 17, 275-290. [CrossRef]

16. Zagar, L.E. The use of the image source method for modeling room acoustics. J. Acoust. Soc. Am. 1983, 74, 1914. [CrossRef]

17. Habets, E. RIR Generator. Available online: https:/ / github.com/ehabets/RIR-Generator (accessed on 25 January 2021).

18. Krokstad, A.; Strom, S.; Sørsdal, S. Calculating the acoustical room response by the use of a ray tracing technique. J. Sound Vib. 1968, 8, 118-125. [CrossRef]

19. Cummings, A. High frequency ray acoustics models for duct silencers. J. Sound Vib. 1999, 221, 681-708. [CrossRef]

20. Lee, J.; Ha, K.; Shung, K.K. A theoretical study of the feasibility of acoustical tweezers: Ray acoustics approach. J. Acoust. Soc. Am. 2005, 117, 3273-3280. [CrossRef] [PubMed]

21. Jensen, F.B.; Kuperman, W.A.; Porter, M.B.; Schmidt, H. Computational Ocean Acoustics, 2nd ed.; Springer: New York, NY, USA, 2011.

22. Petyt, M. Finite Element Techniques for Acoustics. In Theoretical Acoustics and Numerical Techniques; Filippi, P., Ed.; Springer: Vienna, Austria, 1983; pp. 51-103. [CrossRef]

23. Ciskowski, R.D.; Brebbia, C.A. Boundary Element Methods in Acoustics, 1st ed.; Springer: Dordrecht, The Netherlands, 1991.

24. Carotenuto, R.; Pezzimenti, F.; Corte, F.G.D.; Iero, D.; Merenda, M. Acoustic Simulation for Performance Evaluation of Ultrasonic Ranging Systems. Electronics 2021, 10, 1298. [CrossRef]

25. Peebles, P. Probability, Random Variables and Random Signal Principles; Chapter Central Limit Theorem; McGraw-Hill Companies: New York, NY, USA, 2001.

26. Proakis, J.G.; Salehi, M. Digital Communications, 5th ed.; McGraw Hill: Chennai, India, 2008; p. 176.

27. Jain, A.K. Simulation of Indoor Ultrasound Localization System for Assessment of Disruptive Events and Resilience Improvement Options. Master's Thesis, Friedrich Alexander University, Erlangen, Germany, 2018.

28. Damelin, S.B.; Miller, W., Jr. The Mathematics of Signal Processing; Cambridge Texts in Applied Mathematics; Cambridge University Press: Cambridge, UK, 2011. [CrossRef]

29. Adrián-Martínez, S.; Bou-Cabo, M.; Felis, I.; Llorens, C.D.; Martínez-Mora, J.A.; Saldaña, M.; Ardid, M. Acoustic signal detection through the cross-correlation method in experiments with different signal to noise ratio and reverberation conditions. In Ad-Hoc Networks and Wireless; Garcia Pineda, M., Lloret, J., Papavassiliou, S., Ruehrup, S., Westphall, C.B., Eds.; Springer: Berlin/Heidelberg, Germany, 2015; pp. 66-79.

30. Bensky, A. Wireless Positioning Technologies and Applications, 1st ed.; Artech House: London, UK, 2008.

31. Gustafsson, F.; Gunnarsson, F. Positioning using time-difference of arrival measurements. In Proceedings of the 2003 IEEE International Conference on Acoustics, Speech, and Signal Processing, (ICASSP'03), Hong Kong, China, 6-10 April 2003; Volume 6, p. VI-553. [CrossRef]

32. Rocklin, M.; Pinar, A. On Clustering on Graphs with Multiple Edge Types; Taylor and Francis: Oxfordshire, UK, 2011.

33. Branch, M.; Coleman, T.; Li, Y. A subspace, interior, and conjugate gradient method for large-scale bound-constrained minimization problems. SIAM J. Sci. Comput. 1999, 21, 1-23. [CrossRef] 
34. Triggs, B.; McLauchlan, P.; Hartley, R.; Fitzgibbon, A. Bundle adjustment-A modern synthesis. In Vision Algorithms: Theory and Practice; Lecture Notes in Computer Science; Triggs, B., McLauchlan, P., Hartley, R., Eds.; Springer: Berlin/Heidelberg, Germany, 2000; Volume 1883, pp. 298-375. [CrossRef]

35. Thoma, K. Resilience by Design: A Strategy for the Technology Issues of the Future; Acatech-National Academy of Science and Engineering: Munich, Germany, 2014.

36. Panteli, M.; Trakas, D.N.; Mancarella, P.; Hatziargyriou, N.D. Power Systems Resilience Assessment: Hardening and Smart Operational Enhancement Strategies. Proc. IEEE 2017, 105, 1202-1213. [CrossRef]

37. Bruneau, M.; Chang, S.E.; Eguchi, R.T.; Lee, G.C.; O'rourke, T.D.; Reinhorn, A.M.; Shinozuka, M.; Tierney, K.; Wallace, W.A.; von Winterfeldt, D. A Framework to Quantitatively Assess and Enhance the Seismic Resilience of Communities. Earthq. Spectra 2003, 19, 733-752. [CrossRef]

38. Gross, D.; Bolognani, S.; Poolla, B.K.; Dörfler, F. Increasing the Resilience of Low-inertia Power Systems by Virtual Inertia and Damping. In Proceedings of the IREP'2017 Symposium, Espinho, Portugal, 27 August-1 September 2017; p. 64, [CrossRef]

39. Kahan, J.H.; Allen, A.C.; George, J.K. An Operational Framework for Resilience. J. Homel. Secur. Emerg. Manag. 2009, 6, 1-51. [CrossRef]

40. Fafet, C.; Mulolli Zajmi, E. Qualitative Fire Vulnerability Assessments for Museums and Their Collections: A Case Study from Kosovo. Fire 2021, 4, 11. [CrossRef]

41. Amadei, B. A Systems Approach to Building Community Capacity and Resilience. Challenges 2020, 11, 28. [CrossRef]

42. COMSOL Inc. Acoustics Module. Available online: https://www.comsol.com/acoustics-module\#features (accessed on 25 January 2021). 